\title{
An Unusual Cyclopropanation of 9-Bromocamphor Derivatives: A Novel Formal C(1)-C(7) Bond Cleavage of Camphor
}

\author{
Wei-Dong Z. Li* ${ }^{* \dagger *}$ and Yu-Rong Yang ${ }^{\dagger}$ \\ ${ }^{\dagger}$ State Key Laboratory of Applied Organic Chemistry, Lanzhou University, Lanzhou 730000, China; \\ ${ }^{\ddagger}$ State Key Laboratory of Elemento-organic Chemistry, Nankai University, Tianjin 300071, China \\ liwd@1zu.edu.cn; wdli@nankai.edu.cn
}

\section{Experimental}

\section{General}

For product purification by flash column chromatography, silica gel (200 300 mesh) and petroleum ether (bp. 60 90 ${ }^{\circ} \mathrm{C}$ ) are used unless otherwise noted. All solvents were purified and dried by standard techniques, and distilled prior to use. All organic extracts were dried over anhydrous sodium sulfate, unless otherwise noted. IR spectra were recorded on a Nicolet NEXU 670 FT spectrometer as liquid film. ${ }^{1} \mathrm{H}$ and ${ }^{13} \mathrm{C}$ NMR spectra were taken on a Varian Mercury-300, Bruker AM-200 or AM-400 spectrometer with TMS as an internal standard and $\mathrm{CDCl}_{3}$ as solvent. EI-MS was obtained on HP-5988A GC/MS instrument. HRMS were determined on a Bruker Daltonics APEXII 47e FT-ICR spectrometer. Melting points were measured on a Kofler hot stage and are uncorrected. Optical rotations were recorded on a Perkin Elmer 341 polarimeter. All moisture-sensitive reactions were performed in flame-dried glassware under stream of nitrogen. Other commercially available reagents and solvents were used as received without further purification unless indicated otherwise. 


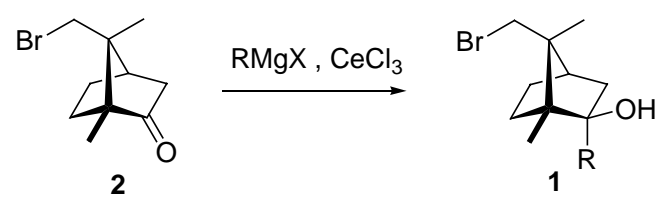

Typical Procedure for the preparation of 9-bromocamphor derivative $1 .^{1}$ $\mathrm{CeCl}_{3} \cdot 7 \mathrm{H}_{2} \mathrm{O}(5.58 \mathrm{~g}, 15.0 \mathrm{mmol})$ was grounded to fine powders in a mortar and transferred into a 250-mL two-necked flask. The flask was immersed in an oil bath and heated gradually to $140-150{ }^{\circ} \mathrm{C}$ under reduced pressure (at $0.2 \mathrm{mmHg}$ ) for $5 \mathrm{~h}$. While the flask was still hot, a stream of argon gas was passed through the flask was then cooled in an ice bath. The resulting anhydrous $\mathrm{CeCl}_{3}$ (fine powder) was treated with THF $(60 \mathrm{~mL})$ and stirred vigorously for $3 \mathrm{~h}$ at room temperature under argon. The flask was immersed in an ice bath, to which a freshly prepared Grignard reagent $(1.0 \mathrm{M}, 15 \mathrm{~mL})$ in THF was added dropwise. After stirring for $1.5 \mathrm{~h}$ at $0{ }^{\circ} \mathrm{C}$, the slurry mixture was treated with a THF $(10$ $\mathrm{mL})$ solution of 9-bromocamphor $2(2.31 \mathrm{~g}, 10.0 \mathrm{mmol})$ and the stirring was continued for $1-2 \mathrm{~h}$. The reaction was then quenched with $10 \%$ aqueous acetic acid carefully, extracted with ether, combined organic extracts were washed with brine, aqueous saturated $\mathrm{NaHCO}_{3}$ solution, brine, and dried. The solvent was removed in vacuum and the residue was purified by chromatography on silica gel to afford the endo-addition product 1.

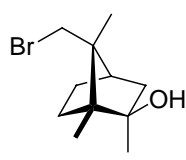

1a, white solids, mp. $79-81^{\circ} \mathrm{C}$; $[\alpha]_{\mathrm{D}}{ }^{21}+4\left(\mathrm{c} 1.6, \mathrm{CHCl}_{3}\right)$; IR (film) $v_{\max } 3464,3410,2963$, 1453, $632 \mathrm{~cm}^{-1}$; ${ }^{1} \mathrm{H}$ NMR (400 MHz, $\mathrm{CDCl}_{3}$ ) $\delta 0.90$ (s, $3 \mathrm{H}$ ), 1.26 (s, $\left.3 \mathrm{H}\right), 1.31$ (s, $3 \mathrm{H}$ ), 1.11-2.09 (m, $8 \mathrm{H}), 3.22(\mathrm{~d}, J=9.9 \mathrm{~Hz}, 1 \mathrm{H}), 3.65(\mathrm{~d}, J=9.9 \mathrm{~Hz}, 1 \mathrm{H}) \mathrm{ppm} ;{ }^{13} \mathrm{C}$ NMR $/ \mathrm{DEPT}\left(100 \mathrm{MHz}, \mathrm{CDCl}_{3}\right) \delta$ $10.4(\mathrm{q}), 17.4(\mathrm{q}), 26.2(\mathrm{t}), 26.8(\mathrm{q}), 31.1(\mathrm{t}), 42.7(\mathrm{t}), 44.0(\mathrm{~d}), 46.5(\mathrm{t}), 53.5(\mathrm{~s}), 53.6(\mathrm{~s}), 80.7(\mathrm{~s}) \mathrm{ppm}$; LRMS (EI) m/z 231 ([M-Me] $\left.]^{+}, 0.2 \%\right), 186$ (3), 108 (77), 95 (37), 93 (32), 67 (24), 43 (100).

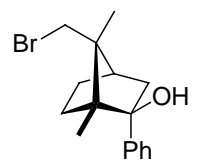

1b, white solids, mp. $116-117^{\circ} \mathrm{C} ;[\alpha]_{\mathrm{D}}^{21}-52\left(\right.$ c 2.0, $\left.\mathrm{CHCl}_{3}\right)$; IR (film) $v_{\max } 3542,3514$, 2949, 1456, 1059, 761, $704 \mathrm{~cm}^{-1} ;{ }^{1} \mathrm{H}$ NMR (400 MHz, $\left.\mathrm{CDCl}_{3}\right) \delta 0.95$ (s, $\left.3 \mathrm{H}\right), 1.49$ (s, $\left.3 \mathrm{H}\right), 1.00-2.42$ (m, $8 \mathrm{H}), 3.23(\mathrm{~d}, J=10 \mathrm{~Hz}, 1 \mathrm{H}), 3.68(\mathrm{~d}, J=10 \mathrm{~Hz}, 1 \mathrm{H}), 7.28-7.55(\mathrm{~m}, 5 \mathrm{H}) \mathrm{ppm} ;{ }^{13} \mathrm{C}$ NMR/DEPT

\footnotetext{
${ }^{1}$ Cf.: (a) Imamoto, T.; Takiyama, N.; Nakamura, K.; Hatajima, T.; Kamiya, Y. J. Am. Chem. Soc. 1989, 111, 4392. (b) Dimitrov, V.; Bratovanov, S.; Simova, S.; Kostova, K. Tetrahedron Lett. 1994, 35, 6713.
} 
$\left(100 \mathrm{MHz}, \mathrm{CDCl}_{3}\right) \delta 10.3(\mathrm{q}), 17.5(\mathrm{q}), 25.9(\mathrm{t}), 31.1(\mathrm{t}), 42.8(\mathrm{t}), 44.2(\mathrm{~d}), 44.6(\mathrm{t}), 54.6(\mathrm{~s}), 54.9(\mathrm{~s})$, 84.6 (s), 126.8 (d), 126.8 (d), 127.2 (d), 127.8 (d), 127.8 (d), 145.3 (s) ppm; LRMS (EI) m/z 229 ([M-Br $\left.]^{+}, 5 \%\right), 211$ (7), 120 (20), 108 (44), 105 (100), 77 (36).

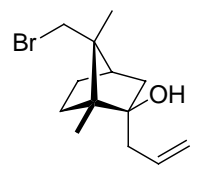

1c, colorless oil, $[\alpha]_{\mathrm{D}}{ }^{21}+34\left(\right.$ c 1.2, $\left.\mathrm{CHCl}_{3}\right)$; IR (film) $v_{\max } 3483,2958 \mathrm{~cm}^{-1} ;{ }^{1} \mathrm{H}$ NMR $\left(200 \mathrm{MHz}, \mathrm{CDCl}_{3}\right) \delta 0.90(\mathrm{~s}, 3 \mathrm{H}), 1.31(\mathrm{~s}, 3 \mathrm{H}), 1.05-2.09$ (m, 8 H), 2.30 (d, J = 7.4 Hz, $\left.2 \mathrm{H}\right), 3.20$ (d, $J=10 \mathrm{~Hz}, 1 \mathrm{H}), 3.65$ (d, $J=10 \mathrm{~Hz}, 1 \mathrm{H}), 5.12-5.22$ (m, 2 H), 5.80-5.97 (m, $1 \mathrm{H}) \mathrm{ppm}$; LRMS (EI) m/z $272\left(\mathrm{M}^{+}, 0.4 \%\right), 231(6), 109(50), 69(74), 41$ (100).

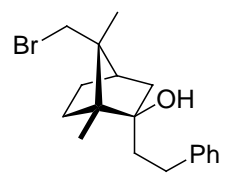

1d, white solids, mp. $89-91{ }^{\circ} \mathrm{C}$; $[\alpha]_{\mathrm{D}}{ }^{21}-11.3$ (c 4.8, $\mathrm{CHCl}_{3}$ ); IR (film) $v_{\max } 3369,2946$ $\mathrm{cm}^{-1} ;{ }^{1} \mathrm{H}$ NMR $\left(200 \mathrm{MHz}, \mathrm{CDCl}_{3}\right) \delta 0.94(\mathrm{~s}, 3 \mathrm{H}), 1.33(\mathrm{~s}, 3 \mathrm{H}), 0.98-2.10(\mathrm{~m}, 10 \mathrm{H}), 2.65-2.82(\mathrm{~m}, 2$ H), 3.20 (d, $J=10 \mathrm{~Hz}, 1 \mathrm{H}), 3.65$ (d, $J=10 \mathrm{~Hz}, 1 \mathrm{H}), 7.28-7.33$ (m, $5 \mathrm{H}) \mathrm{ppm}$; LRMS (EI) m/z 318 $\left(\left[\mathrm{M}-\mathrm{H}_{2} \mathrm{O}\right]^{+}, 0.1 \%\right), 186(8), 108(90), 91$ (100), 79 (16).

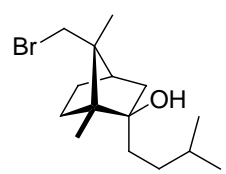

1e, colorless oil, $[\alpha]_{\mathrm{D}}^{21}+31\left(c\right.$ 1.4, $\left.\mathrm{CHCl}_{3}\right)$; IR (film) $v_{\max } 3499,2956,2873,1460,641$ $\mathrm{cm}^{-1}$; ${ }^{1} \mathrm{H}$ NMR (200 MHz, $\left.\mathrm{CDCl}_{3}\right) \delta$ 0.88-0.92 (12 H, $\left.4 \mathrm{Me}\right), 1.00-2.10(\mathrm{~m}, 13 \mathrm{H}), 3.23(\mathrm{~d}, J=10 \mathrm{~Hz}$, $1 \mathrm{H}), 3.70$ (d, $J=10 \mathrm{~Hz}, 1 \mathrm{H})$ ppm; LRMS (EI) m/z $302\left(\mathrm{M}^{+}, 0.2 \%\right), 231$ (4), 186 (8), 166 (8), 108 (100), 93 (32).
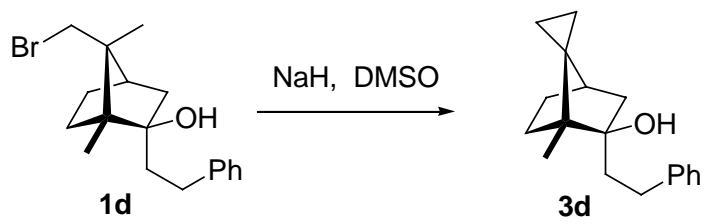

\section{Representative procedure (A) for the spiral-cyclopropanation of 1 with sodium}

hydride. - A mixture of $\mathrm{NaH}$ (dispersion in $60 \%$ oil, $320 \mathrm{mg}, 8.0 \mathrm{mmol}$ ) in DMSO (5 mL) was stirred at $70{ }^{\circ} \mathrm{C}$ for $0.5 \mathrm{~h}$ under Ar atmosphere, to which a solution of $1 \mathbf{d}(1.35 \mathrm{~g}, 4.0 \mathrm{mmol})$ in DMSO $(6 \mathrm{~mL})$ was added dropwise at the same temperature. The resulting reaction mixture was stirred at 70 
${ }^{\circ} \mathrm{C}$ for $1.5 \mathrm{~h}$. After cooling to rt., $15 \mathrm{~mL}$ of water was added and the resulting mixture was extracted thoroughly with ether. The combined organic phases were washed with water, brine, dried, filtered, and concentrated in vacuum. The residue was purified by chromatography on silica gel to afford $0.936 \mathrm{~g}$ $(90 \%)$ of 3d as a colorless oil. $[\alpha]_{\mathrm{D}}{ }^{28}+10\left(c \mathrm{1} .4, \mathrm{CHCl}_{3}\right)$; IR (film) $v_{\max } 3483,3063,3026,2949,1454$, 1004, 749, $698 \mathrm{~cm}^{-1} ;{ }^{1} \mathrm{H}$ NMR (400 MHz, $\left.\mathrm{CDCl}_{3}\right) \delta 0.26(\mathrm{t}, J=7.2 \mathrm{~Hz}, 2 \mathrm{H}), 0.60$ (t, J=7.2 Hz, $2 \mathrm{H}$ ), $0.69(\mathrm{~s}, 3 \mathrm{H}), 1.18-1.93(\mathrm{~m}, 10 \mathrm{H}), 2.70\left(\mathrm{ddd}, J_{1}=4.3 \mathrm{~Hz}, J_{2}=12.6 \mathrm{~Hz}, J_{3}=13.2 \mathrm{~Hz}, 1 \mathrm{H}\right), 2.93$ (ddd, $\left.J_{1}=5.1 \mathrm{~Hz}, J_{2}=12.6 \mathrm{~Hz}, J_{3}=13.2 \mathrm{~Hz}, 1 \mathrm{H}\right), 7.18-7.32(\mathrm{~m}, 5 \mathrm{H}) \mathrm{ppm} ;{ }^{13} \mathrm{C}$ NMR/DEPT $(100 \mathrm{MHz}$, $\left.\mathrm{CDCl}_{3}\right) \delta 0.96(\mathrm{t}), 4.25(\mathrm{t}), 10.2(\mathrm{q}), 28.5(\mathrm{t}), 30.8(\mathrm{t}), 31.1(\mathrm{t}), 36.2(\mathrm{~s}), 38.0(\mathrm{t}), 42.2(\mathrm{~d}), 47.4(\mathrm{t}), 49.1$ (s), 81.7 (s), 125.6 (d), 128.3 (d), 128.3 (d), 128.4 (d), 128.4 (d), 143.4 (s) ppm; LRMS (EI) m/z 256 $\left(\mathrm{M}^{+}, 1 \%\right), 241$ (2), 151 (10), 133 (24), 108 (100), 91 (76); HRMS (ESI) $m / z\left[M-\mathrm{H}_{2} \mathrm{O}+\mathrm{H}\right]^{+}$found 239.1796, calcd 239.1794 for $\mathrm{C}_{18} \mathrm{H}_{23}$.

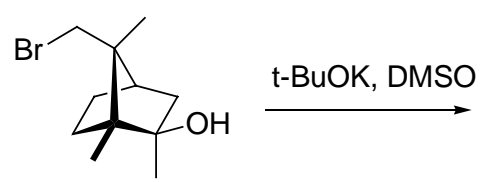

$1 \mathbf{a}$

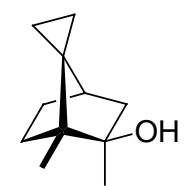

$3 \mathbf{a}$

\section{Representative procedure (B) for the spiral-cyclopropanation of 1 with}

potassium tert-butoxide. - A flask was charged with $t$-BuOK (448 mg, $4.0 \mathrm{mmol}$ ) under Ar, to which was added dropwise a solution of 1a $(494 \mathrm{mg}, 2.0 \mathrm{mmol})$ in DMSO $(6 \mathrm{~mL})$ with stirring at $30^{\circ} \mathrm{C}$. The resulting mixture was continued to stir at $40{ }^{\circ} \mathrm{C}$ for $6 \mathrm{~h}$ and monitored by TLC. After cooling to rt, $10 \mathrm{~mL}$ of water was added and the resulting mixture was extracted thoroughly with ether. The combined organic phases were washed with water, brine, dried, filtered, and the solvent removed in vacuum. The residue was purified by chromatography on silica gel to afford $280 \mathrm{mg}(85 \%)$ of $\mathbf{3 a}$ and 3a' (ca. 5\%) as white solids. 3a, mp. 80-82 ${ }^{\circ} \mathrm{C}$; $[\alpha]_{\mathrm{D}}{ }^{28}+17.8$ (c 4.4, $\mathrm{CHCl}_{3}$ ); IR (film) $v_{\max } 3408,3066$, 2949, 2868, 1099, $1064 \mathrm{~cm}^{-1}$; ${ }^{1} \mathrm{HNMR}\left(200 \mathrm{MHz}, \mathrm{CDCl}_{3}\right) \delta 0.27(\mathrm{t}, J=6.8 \mathrm{~Hz}, 2 \mathrm{H}), 0.55(\mathrm{t}, J=6.8$ $\mathrm{Hz}, 2 \mathrm{H}), 0.66$ (s, $3 \mathrm{H}), 1.22$ (s, $3 \mathrm{H}), 0.88-1.81$ (m, $8 \mathrm{H}) \mathrm{ppm} ;{ }^{13} \mathrm{C}$ NMR/DEPT $\left(50 \mathrm{MHz}, \mathrm{CDCl}_{3}\right) \delta$ $1.04(\mathrm{t}), 4.05(\mathrm{t}), 9.52(\mathrm{q}), 22.1(\mathrm{q}), 28.3(\mathrm{t}), 31.7(\mathrm{t}), 35.8(\mathrm{~s}), 42.4(\mathrm{~d}), 48.5(\mathrm{~s}), 49.3(\mathrm{t}), 79.9(\mathrm{~s}) \mathrm{ppm}$; HRMS (SMS) $m / z$ [M- $\left.\mathrm{H}_{2} \mathrm{O}+\mathrm{H}\right]^{+}$found 149.1325 , calcd 149.1325 for $\mathrm{C}_{11} \mathrm{H}_{17}$. 


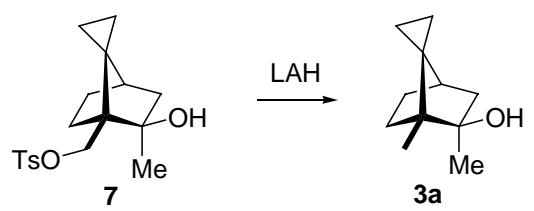

Alternative preparation of 3a by LAH reductive deoxygenation of tosylate 7. - To a stirred solution of tosylate $7^{2}$ (mixture of isomers at $\mathrm{C}(2), 140 \mathrm{mg}, 0.42 \mathrm{mmol}$ ) in anhydrous ether (10 $\left.\mathrm{mL}\right)$ was added $\mathrm{LiAlH}_{4}(100 \mathrm{mg}, 2.6 \mathrm{mmol})$ in one portion. The reaction mixture was brought to reflux for $2 \mathrm{~h}$, cooled to room temperature, and quenched with dilute aqueous $\mathrm{NaOH}$. The mixture was extracted with ether, dried over anhydrous sodium sulfate, filtered, and concentrated. Chromatography on silica gel of the crude residue provided $30 \mathrm{mg}(42 \%)$ of $3 \mathbf{a}$ (spectroscopically identical with the above obtained 3a) along with $15 \mathrm{mg}(22 \%)$ of its $\mathrm{C}(2)$ isomer.

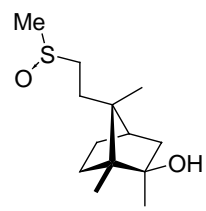

3a', yield 5\%, white solids (mixture of isomers, ca. $1: 1$ ), mp. $155-157^{\circ} \mathrm{C}$, IR (film) $v_{\max }$ 3385, 2957, 2874, 1453, 1105, $1026 \mathrm{~cm}^{-1} ;{ }^{1} \mathrm{H}$ NMR (200 MHz, $\left.\mathrm{CDCl}_{3}\right) \delta 0.89$ (s, $\left.3 \mathrm{H}\right), 1.17$ (s, $\left.3 \mathrm{H}\right)$, $1.27(\mathrm{~s}, 3 \mathrm{H}), 1.10-2.08(\mathrm{~m}, 8 \mathrm{H}), 2.58(\mathrm{~s}, 3 \mathrm{H}), 2.56-2.80(\mathrm{~m}, 2 \mathrm{H}) \mathrm{ppm} ;{ }^{13} \mathrm{C}$ NMR/DEPT $(50 \mathrm{MHz}$, $\left.\mathrm{CDCl}_{3}\right) \delta 10.0(\mathrm{q}), 10.0(\mathrm{q}), 17.4(\mathrm{q}), 17.6(\mathrm{q}), 25.9(\mathrm{t}), 26.1(\mathrm{t}), 26.3(\mathrm{t}), 26.5(\mathrm{t}), 27.1(\mathrm{q}), 27.1(\mathrm{q}), 30.9$ $(\mathrm{t}), 30.9(\mathrm{t}), 38.2(\mathrm{q}), 38.4(\mathrm{q}), 42.3(\mathrm{~d}), 42.4(\mathrm{~d}), 46.7(\mathrm{t}), 46.7(\mathrm{t}), 50.7(\mathrm{t}), 51.3(\mathrm{t}), 51.4(\mathrm{~s}), 51.4(\mathrm{~s})$, $53.0(\mathrm{~s}), 53.0(\mathrm{~s}), 79.3(\mathrm{~s}), 79.3(\mathrm{~s}) \mathrm{ppm}$; HRMS (ESI) $\mathrm{m} / \mathrm{z}[\mathrm{M}+\mathrm{H}]^{+}$found 245.1565, calcd 245.1470 for $\mathrm{C}_{13} \mathrm{H}_{25} \mathrm{O}_{2} \mathrm{~S}$.

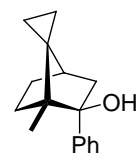

3b, colorless oil, $[\alpha]_{\mathrm{D}}{ }^{21}-27$ (c 2.6, $\mathrm{CHCl}_{3}$ ); IR (film) $v_{\max } 3452,3060,2958,1450,1060,759$, $703 \mathrm{~cm}^{-1} ;{ }^{1} \mathrm{H}$ NMR $\left(200 \mathrm{MHz}, \mathrm{CDCl}_{3}\right) \delta 0.33(\mathrm{t}, J=7.4 \mathrm{~Hz}, 2 \mathrm{H}), 0.62(\mathrm{~s}, 3 \mathrm{H}), 0.72(\mathrm{t}, J=7.4 \mathrm{~Hz}, 2$ $\mathrm{H}), 1.17-2.46(\mathrm{~m}, 8 \mathrm{H}), 7.28-7.51(\mathrm{~m}, 5 \mathrm{H}) \mathrm{ppm} ;{ }^{13} \mathrm{C}$ NMR/DEPT $\left(50 \mathrm{MHz}, \mathrm{CDCl}_{3}\right) \delta 1.68(\mathrm{t}), 4.52(\mathrm{t})$, $9.48(\mathrm{q}), 28.2(\mathrm{t}), 31.2(\mathrm{t}), 37.5(\mathrm{~s}), 42.6(\mathrm{~d}), 48.2(\mathrm{t}), 50.5(\mathrm{~s}), 83.8(\mathrm{~s}), 126.0(\mathrm{~d}), 126.8(\mathrm{~d}), 126.8(\mathrm{~d})$, 127.4 (d), 127.4 (d), 142.5 (s) ppm; LRMS (EI) m/z $228\left(\mathrm{M}^{+}, 0.7 \%\right), 213$ (4), 210 (18), 108 (97), 105 (60), 93 (100), 79 (86), 51 (26).

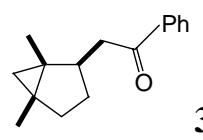

3b', colorless oil, $[\alpha]_{\mathrm{D}}{ }^{21}+50\left(c 1.7, \mathrm{CHCl}_{3}\right)$; IR (film) $v_{\max } 2953,2864,1684,1596,1449$,

\footnotetext{
${ }^{2}$ Prepared from 10-hydroxy-7-spiral-cyclopropylcamphor by methylation with MeLi and tosylation (TsCl, Pyr.) in moderate yield, cf.: Föhlisch, B.; Bakr, D. A.; Fischer, P. J. Org. Chem. 2002, 67, 3682.
} 
1274, $754 \mathrm{~cm}^{-1}$; ${ }^{1} \mathrm{H}$ NMR $\left(300 \mathrm{MHz}, \mathrm{CDCl}_{3}\right) \delta-0.08(\mathrm{~d}, J=4.2 \mathrm{~Hz}, 1 \mathrm{H}), 0.57(\mathrm{~d}, J=4.5 \mathrm{~Hz}, 1 \mathrm{H})$, 1.09 (s, $3 \mathrm{H}), 1.16$ (s, $3 \mathrm{H}), 1.18-1.33$ (m, $2 \mathrm{H}), 1.64-1.69$ (m, 2 H), 2.60-2.68 (m, $2 \mathrm{H}), 3.13-3.22$ (m, $1 \mathrm{H}), 7.42-7.96$ (m, $5 \mathrm{H}) \mathrm{ppm} ;{ }^{13} \mathrm{C} \mathrm{NMR} / \mathrm{DEPT}\left(75 \mathrm{MHz}, \mathrm{CDCl}_{3}\right.$ ) $\delta 15.7$ (q), 18.1 (q), 20.9 (t), 25.7 (t), $27.5(\mathrm{~s}), 29.5$ (s), 32.6 (t), 40.8 (d), 41.2 (t), 128.0 (d), 128.0 (d), 128.5 (d), 128.5 (d), 132.7 (d), 137.3 (s), 200.6 (s) ppm; LRMS (EI) m/z 228 (M+1, 0.5\%), 121 (3), 108 (100), 105 (64), 93 (60), 77 (40); HRMS (ESI) $m / z$ [M+H] found 229.1588, calcd 229.1587 for $\mathrm{C}_{16} \mathrm{H}_{21} \mathrm{O}$.

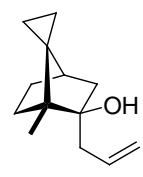

3c, colorless oil, $[\alpha]_{\mathrm{D}}^{28}+24\left(\right.$ c 1.0, $\left.\mathrm{CHCl}_{3}\right)$; IR (film) $v_{\max } 3481,3069,2950,2872,1639$, 1456, 1065, 988, $910 \mathrm{~cm}^{-1} ;{ }^{1} \mathrm{H}$ NMR (200 MHz, $\left.\mathrm{CDCl}_{3}\right) \delta 0.22$ (t, J=7.0 Hz, $\left.2 \mathrm{H}\right), 0.56$ (t, J= 7.0 Hz, $2 \mathrm{H}), 0.67$ (s, $3 \mathrm{H}), 1.17-1.77$ (m, 8 H), 2.29 (t, $J=6.0 \mathrm{~Hz}, 2 \mathrm{H}), 5.08-5.16(\mathrm{~m}, 2 \mathrm{H}), 5.93-6.02(\mathrm{~m}, 1$ H) ppm; ${ }^{13} \mathrm{C}$ NMR/DEPT (50 MHz, CDCl $) \delta 1.02(\mathrm{t}), 4.30(\mathrm{t}), 10.3(\mathrm{q}), 28.3(\mathrm{t}), 31.3(\mathrm{t}), 36.2(\mathrm{~s}), 40.8$ (t), 42.2 (d), 47.2 (t), 48.9 (s), 80.9 (s), 117.2 (t), 135.2 (d) ppm; LRMS (EI) m/z $192\left(\mathrm{M}^{+}, 0.1 \%\right), 177$ (1), 151 (29), 109 (69), 108 (88), 93 (100), 77 (44).

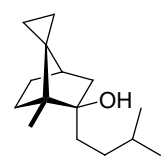

3e, colorless oil, $[\alpha]_{\mathrm{D}}{ }^{28}+16\left(\right.$ c 1.2, $\left.\mathrm{CHCl}_{3}\right)$; IR (film) $v_{\max } 3484,3065,2953,1464 \mathrm{~cm}^{-1} ;{ }^{1} \mathrm{H}$ NMR (200 MHz, $\left.\mathrm{CDCl}_{3}\right) \delta 0.22(\mathrm{t}, J=7.1 \mathrm{~Hz}, 2 \mathrm{H}), 0.56$ (t, $\left.J=7.1 \mathrm{~Hz}, 2 \mathrm{H}\right), 0.66$ (s, $\left.3 \mathrm{H}\right), 0.90$ (d, $J=$ $6.2 \mathrm{~Hz}, 6 \mathrm{H}), 1.16-1.68(\mathrm{~m}, 13 \mathrm{H}) \mathrm{ppm} ;{ }^{13} \mathrm{C} \mathrm{NMR} / \mathrm{DEPT}\left(50 \mathrm{MHz}, \mathrm{CDCl}_{3}\right) \delta 0.86(\mathrm{t}), 4.20(\mathrm{t}), 10.2(\mathrm{q})$, $22.6(\mathrm{q}), 22.7(\mathrm{q}), 28.5(\mathrm{t}), 28.7(\mathrm{~d}), 31.1(\mathrm{t}), 33.1(\mathrm{t}), 33.3(\mathrm{t}), 36.1(\mathrm{~s}), 42.1(\mathrm{~d}), 47.3(\mathrm{t}), 48.9(\mathrm{~s}), 81.8$ (s) ppm; LRMS (EI) m/z 222 (M+, $0.1 \%), 207$ (1), 133 (12), 108 (100), 93 (90), 79 (84); HRMS (ESI) $m / z\left[M-\mathrm{H}_{2} \mathrm{O}+\mathrm{H}\right]^{+}$found 205.1956, calcd 205.1951 for $\mathrm{C}_{15} \mathrm{H}_{25}$.

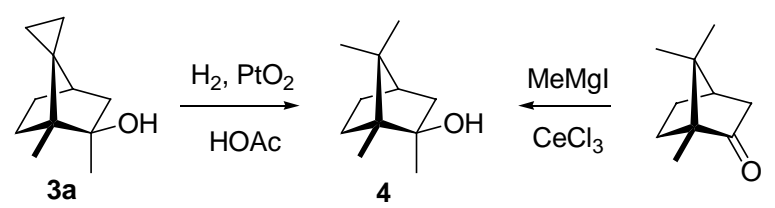

Hydrogenolysis of spiral-cyclopropyl camphor 3a. - A solution of spiral-cyclopropyl camphor derivative 3a $(56 \mathrm{mg}, 0.34 \mathrm{mmol})$ in glacial acetic acid $(2 \mathrm{~mL})$ was charged with platinum oxide (6 mg) and stirred under hydrogen atmosphere (balloon pressure) for $2 \mathrm{~h}$. The mixture was diluted with sat. $\mathrm{NaHCO}_{3}$ and extracted with ether, washed with sat. $\mathrm{NaHCO}_{3}$, brine, dried, filtered, and concentrated in vacuum. The residue was purified by chromatography to afford 4 (44 mg, yield 
$80 \%$ ) as white solids, which is identical spectroscopically with the organocerium methylation adduct of D-camphor as shown above. Mp. $86-88^{\circ} \mathrm{C} ;[\alpha]_{\mathrm{D}}{ }^{21}-5\left(\right.$ c 2.2, $\left.\mathrm{CHCl}_{3}\right){ }^{3}$ IR (film) $v_{\max } 3469,2947,1452$, 1371, $1094 \mathrm{~cm}^{-1} ;{ }^{1} \mathrm{H}$ NMR (200 MHz, $\left.\mathrm{CDCl}_{3}\right) \delta 0.84$ (s, $\left.3 \mathrm{H}\right), 0.86$ (s, $\left.3 \mathrm{H}\right), 1.10$ (s, $\left.3 \mathrm{H}\right), 1.24(\mathrm{~s}, 3 \mathrm{H})$ ppm; LRMS (EI) m/z 168 (M+, 3\%), 150 (4), 108 (25), 95 (100), 43 (41).

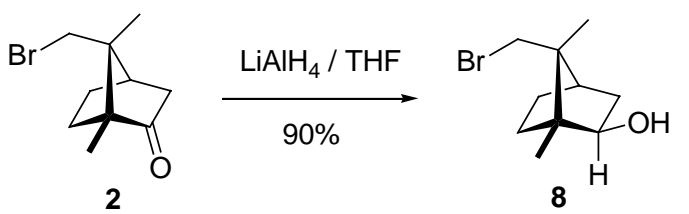

Preparation of exo-9-Bromobornan-2-ol (8). - To a suspension of lithium aluminum hydride (120 $\mathrm{mg}, 3.2 \mathrm{mmol})$ in $4 \mathrm{~mL}$ of THF was added dropwise a solution of $1.0 \mathrm{~g}(4.3 \mathrm{mmol})$ of 9-bromocamphor (2) in $4 \mathrm{~mL}$ of THF at $-78^{\circ} \mathrm{C}$. The mixture was stirred at same temperature for an additional hour. The reaction was quenched with $1.5 \mathrm{~mL}$ of aqueous $\mathrm{NaOH}(2.0 \mathrm{M})$. The crude product was diluted with ether, filtered, dried, and concentrated. The resulting solid residue was recrystallized from hexane to give $900 \mathrm{mg}$ (yield 90\%) of exo-9-bromobornan-2-ol (8). Mp. 105-107 ${ }^{\circ} \mathrm{C}\left[\text { lit. } 105-107^{\circ} \mathrm{C}\right]^{4} ;[\alpha]_{\mathrm{D}}{ }^{21}-3$ (c $0.04, \mathrm{CHCl}_{3}$ ); IR (film) $v_{\max } 3389,2954,2883,1454,1241,1068,1001,642 \mathrm{~cm}^{-1} ;{ }^{1} \mathrm{H}$ NMR (300 $\left.\mathrm{MHz}, \mathrm{CDCl}_{3}\right) \delta 0.95(\mathrm{~s}, 3 \mathrm{H}), 1.22(\mathrm{~s}, 3 \mathrm{H}), 1.04-1.85(\mathrm{~m}, 7 \mathrm{H}), 2.08(\mathrm{~s}, 1 \mathrm{H}), 3.19(\mathrm{~d}, J=9.9 \mathrm{~Hz}, 1 \mathrm{H})$, $3.52(\mathrm{~d}, J=9.9 \mathrm{~Hz}, 1 \mathrm{H}), 3.71(\mathrm{~s}, 1 \mathrm{H}) \mathrm{ppm} ;{ }^{13} \mathrm{C} \mathrm{NMR}\left(75 \mathrm{MHz}, \mathrm{CDCl}_{3}\right) \delta$ 11.7, 16.2, 26.4, 33.6, 39.4, 42.4, 43.4, 50.3, 51.2, 81.0 ppm; LRMS (EI) m/z 232 (M+1, 0.1\%), 173 (5), 153 (47), 135 (37), 107 (42), 95 (100), 79 (31), 69 (63).

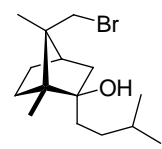

9, colorless oil; IR (film) $v_{\max } 3448,2952,2869,1463,1015 \mathrm{~cm}^{-1}$; ${ }^{1} \mathrm{H}$ NMR $(200 \mathrm{MHz}$, $\left.\mathrm{CDCl}_{3}\right) \delta$ 0.79-0.91 (m, $\left.12 \mathrm{H}, 4 \mathrm{Me}\right), 1.01-2.05(\mathrm{~m}, 13 \mathrm{H}), 3.41(\mathrm{~d}, J=7.8 \mathrm{~Hz}, 1 \mathrm{H}), 3.65(\mathrm{~d}, J=7.8$ $\mathrm{Hz}, 1 \mathrm{H}) \mathrm{ppm}$.

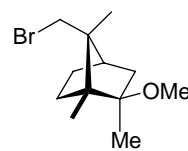

10, colorless oil; IR (film) $v_{\max } 2964,2867,1453,1122,1065 \mathrm{~cm}^{-1} ;{ }^{1} \mathrm{H}$ NMR $(80 \mathrm{MHz}$, $\left.\mathrm{CDCl}_{3}\right) \delta 0.89(\mathrm{~s}, 3 \mathrm{H}), 1.15(\mathrm{~s}, 3 \mathrm{H}), 1.21(\mathrm{~s}, 3 \mathrm{H}), 3.12(\mathrm{~s}, 3 \mathrm{H}), 0.94-2.11(\mathrm{~m}, 7 \mathrm{H}), 3.22(\mathrm{~d}, J=10 \mathrm{~Hz}$,

\footnotetext{
${ }^{3}$ Cf.: (a) Dictionary of Natural Products; Buckingham, J. Eds.; Chapman \& Hall, 1994; Vol. 4, p. 3890. (b) Toivonen, H. Tetrahedron Lett. 1968, 26, 3041.

${ }^{4}$ Cf.: Vaillancourt, V.; Agharahimi, M. R.; Sundram, U. N.; Richou, O.; Faulkner, D. J.; Albizati, K. F. J. Org. Chem. 1991, 56, 378 .
} 
$1 \mathrm{H}), 3.71(\mathrm{~d}, J=10 \mathrm{~Hz}, 1 \mathrm{H})$; LRMS (EI) m/z $260\left(\mathrm{M}^{+}, 0.1 \%\right), 186$ (8), 149 (12), 121 (16), 109 (76), 95 (39), 79 (22), 43 (94), 41 (100).
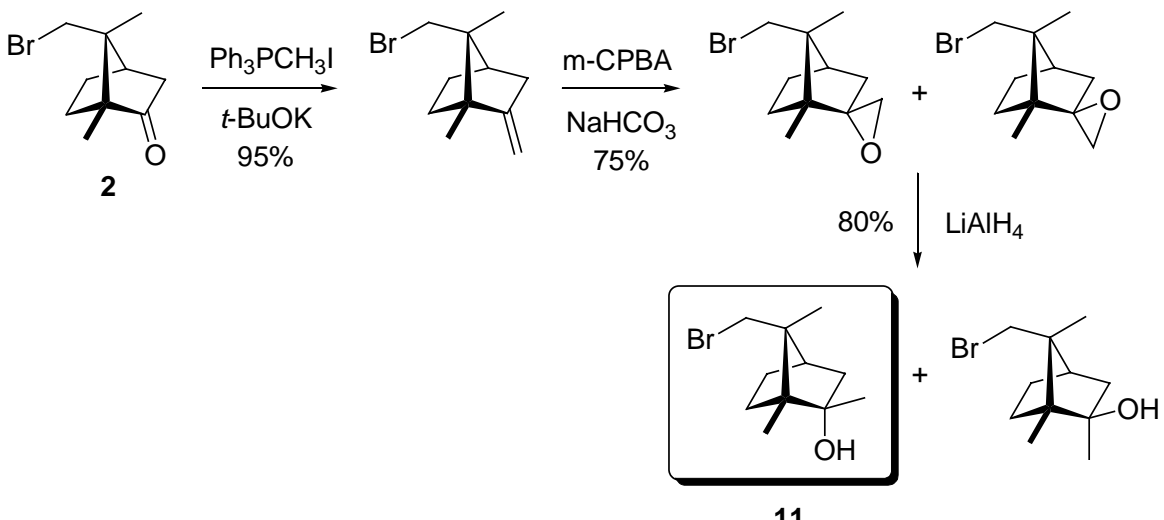

11

Preparation of compound 11. - (a) methylenation ${ }^{5}$ of 2. - To a stirred suspension of $t$-BuOK (784 $\mathrm{mg}, 7.0 \mathrm{mmol})$ in anhydrous benzene $(15 \mathrm{~mL})$ under Ar was added methyltriphenylphosphorium iodide powder $(2.82 \mathrm{~g}, 7 \mathrm{mmol})$ and the yellowish mixture was heated to reflux for $1 \mathrm{~h}$ followed by evaporation of the solvent under a stream of $\mathrm{Ar}$ at ca. $90-100{ }^{\circ} \mathrm{C}$, until a slurry remained. To which was then added dropwise a mixture of 9-bromocamphor $(1.15 \mathrm{~g}, 5.0 \mathrm{mmol})$ and benzene $(2 \mathrm{~mL})$ at the same temperature under Ar. After stirring for 10 minutes, the reaction mixture was cooled to room temperature and was diluted with water $(15 \mathrm{~mL})$, extracted with ether. The combined organic phases were washed with water, brine, dried over $\mathrm{MgSO}_{4}$, filtered, and concentrated. The residue was purified by chromatography on silica gel to afford $1.08 \mathrm{~g}(95 \%)$ of the olefin as a colorless oil. IR (film) $v_{\max }$ 2957, 2877, 1658, 1445, 1379, 1245, 879, $631 \mathrm{~cm}^{-1} ;{ }^{1} \mathrm{H}$ NMR (300 MHz, $\left.\mathrm{CDCl}_{3}\right) \delta 0.95$ (s, $\left.6 \mathrm{H}, 2 \mathrm{Me}\right)$, 1.26-2.39 (m, 7H), $3.22(\mathrm{~d}, J=9.9 \mathrm{~Hz}, 1 \mathrm{H}), 3.62(\mathrm{~d}, J=9.9 \mathrm{~Hz}, 1 \mathrm{H}), 4.67(\mathrm{~d}, J=14 \mathrm{~Hz}, 2 \mathrm{H}) \mathrm{ppm}$; ${ }^{13} \mathrm{C}$ NMR $\left(75 \mathrm{MHz}, \mathrm{CDCl}_{3}\right) \delta 12.7,16.2,27.2,34.7,36.0,41.0,42.8,51.9,52.6,101.7,158.7$ ppm; EIMS m/z 228 (M+1 1\%), 149 (94), 121 (16), 107 (97), 93 (100), 79 (47), 41 (53). (b) Preparation of epoxide intermediates. To a solution of the above olefin $(230 \mathrm{mg}, 1.0 \mathrm{mmol})$ in $\mathrm{CH}_{2} \mathrm{Cl}_{2}(3 \mathrm{~mL})$ was added $m$-CPBA (350 mg, $1.4 \mathrm{mmol}, 70 \%)$ and $\mathrm{NaHCO}_{3}(840 \mathrm{mg}, 10.0 \mathrm{mmol})$ with stirring at $0{ }^{\circ} \mathrm{C}$. The resulting mixture was stirred for $1 \mathrm{~h}$ then quenched with aqueous $\mathrm{Na}_{2} \mathrm{SO}_{3}$, extracted with $\mathrm{CH}_{2} \mathrm{Cl}_{2}$ and the organic phases were washed with sat. $\mathrm{NaHCO}_{3}$, brine, dried over $\mathrm{MgSO}_{4}$, filtered, and concentrated in vacuum. The residue was purified by chromatography on silica gel to afford $180 \mathrm{mg}(75 \%)$ of the

${ }^{5}$ Cf.: Fitjer, L.; Quabeck, U. Synth. Commun. 1985, 15, 855. 
epoxides as a colorless oil (mixture of diastereomers as shown). IR (film) $v_{\max } 2959,2880,1450,1380$, 1248, 1037, 799, $626 \mathrm{~cm}^{-1} ;{ }^{1} \mathrm{H}$ NMR $\left(300 \mathrm{MHz}, \mathrm{CDCl}_{3}\right) \delta 0.65$ (s, $\left.6 \mathrm{H}, 2 \mathrm{Me}\right), 1.14(\mathrm{~s}, 3 \mathrm{H}), 1.18(\mathrm{~s}, 3$ H), 1.30-2.23 (m, $14 \mathrm{H}), 2.61(\mathrm{~d}, J=4.2 \mathrm{~Hz}, 1 \mathrm{H}), 2.66(\mathrm{~d}, J=4.5 \mathrm{~Hz}, 2 \mathrm{H}), 2.87$ (d, $J=4.5 \mathrm{~Hz}, 1 \mathrm{H})$, $3.22(\mathrm{~d}, J=9.9 \mathrm{~Hz}, 2 \mathrm{H}), 3.54(\mathrm{~d}, J=10.2 \mathrm{~Hz}, 1 \mathrm{H}), 3.61(\mathrm{~d}, J=10.5 \mathrm{~Hz}, 1 \mathrm{H}) \mathrm{ppm} ;{ }^{13} \mathrm{C}$ NMR $(75 \mathrm{MHz}$, $\left.\mathrm{CDCl}_{3}\right) \delta 8.85,10.5,16.0,16.1,26.6,26.9,30.2,32.2,35.6,36.9,40.6,40.9,42.7,42.9,46.7,48.3$, 48.7, 52.3, 52.4, 54.0, 68.5, 68.5 ppm; LRMS (EI) m/z 244 (M+, 0.1\%), 165 (90), 135 (20), 121 (17), 107 (69), 93 (85), 91 (60), 79 (52). (c) Preparation of 11. To a suspension of lithium aluminum hydride (30 mg, $0.8 \mathrm{mmol})$ in THF ( $1 \mathrm{~mL}$ ) was added a solution of the above epoxides (100 $\mathrm{mg}, 0.41$ mmol) in THF $(1 \mathrm{~mL})$. After the reaction mixture was stirred for 10 minutes under refluxing and cooled to ambient temperature, saturated aqueous sodium sulfate was added. The crude product was filtered through a short pad of Celite with diethyl ether, the filtrate was concentrated, and then purified by chromatography on silica gel to give $50 \mathrm{mg}(50 \%)$ of the endo- hydroxyl product 11 along with $30 \mathrm{mg}$ (30\%) of 1a. 11, white solids, mp. $96-98{ }^{\circ} \mathrm{C}$; $[\alpha]_{\mathrm{D}}{ }^{21}-17.2\left(\right.$ (c $\left.4.7 \mathrm{CHCl}_{3}\right)$; IR (film) $v_{\max } 3403,2953$, 1450, 1115, 1071, 731, $703 \mathrm{~cm}^{-1} ;{ }^{1} \mathrm{H}$ NMR (300 MHz, $\left.\mathrm{CDCl}_{3}\right) \delta 0.84$ (s, $\left.3 \mathrm{H}\right), 1.13$ (s, $\left.3 \mathrm{H}\right), 1.33$ (s, 3 H), 1.12-1.76 (m, 5 H), 1.99-2.02 (m, $2 \mathrm{H}), 2.23-2.35$ (m, $1 \mathrm{H}), 3.20$ (d, $J=10.2 \mathrm{~Hz}, 1 \mathrm{H}), 3.73$ (d, $J$ $=10 \mathrm{~Hz}, 1 \mathrm{H}) \mathrm{ppm} ;{ }^{13} \mathrm{C} \mathrm{NMR}\left(75 \mathrm{MHz}, \mathrm{CDCl}_{3}\right) \delta 11.6,17.5,26.4,29.1,29.3,43.0,43.6,45.8,53.6$, 54.0, 79.5 ppm; LRMS (EI) m/z 231 ([M-Me] , 0.4\%), 186 (6), 108 (95), 95 (39), 43 (100).

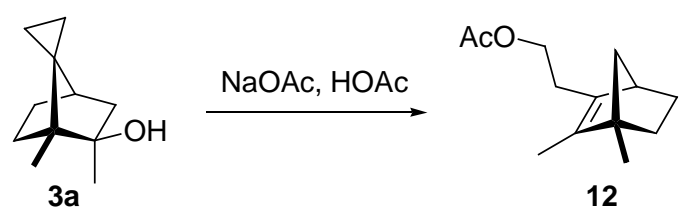

Preparation of acetate 12a $(X=\mathbf{O A c}, \mathbf{R}=\mathbf{M e})$. - To a solution of spiral-cyclopropyl camphor derivative $3 \mathbf{a}(50 \mathrm{mg}, 0.3 \mathrm{mmol})$ in glacial acetic acid $(1 \mathrm{~mL})$ was added sodium acetate $(246 \mathrm{mg}, 3.0$ $\mathrm{mmol}$ ), and the mixture was heated to reflux with stirring for $2 \mathrm{~h}$. After cooling to rt., the reaction mixture was neutralized with powdered $\mathrm{NaHCO}_{3}$, diluted with ether, washed with sat. aqueous $\mathrm{NaHCO}_{3}$, water, and brine respectively, dried over $\mathrm{MgSO}_{4}$, filtered and concentrated in vacuum. The residue was purified by chromatography on silica gel to afford the norborenyl acetate 12a as a colorless oil. $[\alpha]_{\mathrm{D}}{ }^{21}-18\left(\right.$ ( $\left.1.4, \mathrm{CHCl}_{3}\right)$; IR (film) $v_{\max } 2952,1743,1243,1039 \mathrm{~cm}^{-1} ;{ }^{1} \mathrm{H}$ NMR $\left(200 \mathrm{MHz}, \mathrm{CDCl}_{3}\right)$ $\delta$ 1.00-2.42 (m, 8 H), 1.22 (s, 3 H), 1.53 (s, 3 H), 2.03 (s, 3 H), 2.62 (brs, $1 \mathrm{H}), 4.08$ (t, J = 7.4 Hz, 2 H) ppm; ${ }^{13} \mathrm{C}$ NMR/DEPT (50 MHz, CDCl $) \delta 8.87$ (q), $17.6(q), 20.9$ (q), 26.9 (t), $28.9(\mathrm{t}), 32.6(\mathrm{t}), 44.9$ 
(d), $51.6(\mathrm{~s}), 53.3(\mathrm{t}), 63.3(\mathrm{t}), 136.0$ (s), 140.3 (s), 171.0 (s) ppm; LRMS (EI) m/z $208\left(\mathrm{M}^{+}, 0.3 \%\right), 148$ (20), 120 (100), 105 (70), 91 (30); HRMS (ESI) $\mathrm{m} / \mathrm{z}[\mathrm{M}+\mathrm{H}]^{+}$found 209.1537, calcd 209.1536 for $\mathrm{C}_{13} \mathrm{H}_{21} \mathrm{O}_{2}$.

$\mathrm{AcO}$<smiles>CCCC1CCCC1(CC)CCC</smiles>

12b $(X=$ OAc, $R=P h)$, colorless oil; IR (film) $v_{\max } 2956,1741,1239,1231,1034,760$, $700 \mathrm{~cm}^{-1} ;{ }^{1} \mathrm{H}$ NMR $\left(200 \mathrm{MHz}, \mathrm{CDCl}_{3}\right) \delta 1.29(\mathrm{~s}, 3 \mathrm{H}), 2.05$ (s, $\left.3 \mathrm{H}\right), 1.22-1.95(\mathrm{~m}, 6 \mathrm{H}), 2.40(\mathrm{~m}, 2 \mathrm{H})$,

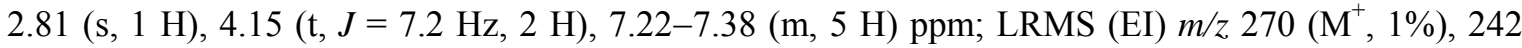
(12), 210 (24), 182 (100), 167 (86), 43 (80).

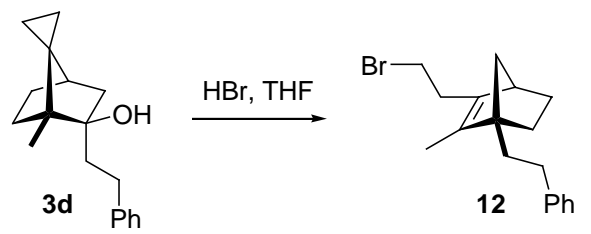

Preparation of bromide 12d $(X=B r, R=$ phenethyl $)$. - To a stirred solution of spiral-cyclopropyl camphor derivative $3 \mathbf{d}$ (380 $\mathrm{mg}, 1.48 \mathrm{mmol})$ in $4 \mathrm{~mL}$ of THF was added dropwise $5 \mathrm{~mL}$ of $\mathrm{HBr}(40 \%$ aqueous) at $0{ }^{\circ} \mathrm{C}$ and the resulting mixture was stirred for $10 \mathrm{~min}$, then quenched and neutralized with sat. aqueous $\mathrm{NaHCO}_{3}$. The aqueous phase was separated and extracted with ether. The combined organic phases were washed with brine, dried over $\mathrm{MgSO}_{4}$, filtered, and concentrated. The residue was purified by chromatography on silica gel to afford $400 \mathrm{mg}(85 \%)$ of the norborenyl bromide 12d as a colorless oil. $[\alpha]_{\mathrm{D}}{ }^{21}-21\left(\mathrm{c} 1.5, \mathrm{CHCl}_{3}\right) ;{ }^{1} \mathrm{H} \mathrm{NMR}\left(200 \mathrm{MHz}, \mathrm{CDCl}_{3}\right) \delta 0.95-1.28$ (m, $\left.4 \mathrm{H}\right), 1.61$ (s, 3 H), 1.72-2.03 (m, 4 H), 2.56-2.74 (m, 5 H), 3.42 (t, $J=7.8 \mathrm{~Hz}, 2 \mathrm{H}), 7.18-7.33$ (m, 5 H) ppm; LRMS (EI) m/z $318\left(\mathrm{M}^{+}, 1 \%\right), 290$ (13), 239 (10), 199 (100), 119 (23), 91 (77), 77 (15).

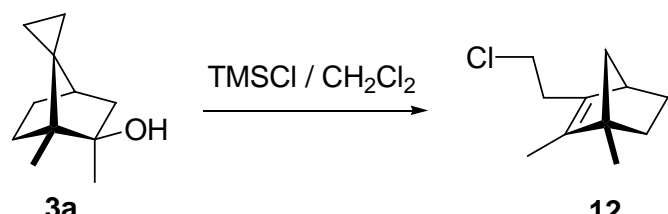

Preparation of chloride 12a' $(\mathrm{X}=\mathbf{C l}, \mathbf{R}=\mathbf{M e})$. - To a solution of spiral-cyclopropyl camphor derivative $3 a$ ( $20 \mathrm{mg}, 0.12 \mathrm{mmol})$ in $\mathrm{CH}_{2} \mathrm{Cl}_{2}(1 \mathrm{~mL})$ was added freshly distilled TMSCl $(0.2 \mathrm{~mL})$ at $-15{ }^{\circ} \mathrm{C}$ with stirring and the reaction mixture was stirred for 30 minutes, then quenched with sat. aqueous $\mathrm{NaHCO}_{3}$. The mixture was extracted with ether, washed with sat. aqueous $\mathrm{NaHCO}_{3}$, brine, 
dried over $\mathrm{MgSO}_{4}$, filtered, and concentrated in vacuum. The residue was purified by chromatography on silica gel to afford $13 \mathrm{mg}(60 \%)$ of the chloride 12a' as a colorless oil. $[\alpha]_{\mathrm{D}}{ }^{18}-23\left(c 0.6, \mathrm{CHCl}_{3}\right)$; IR (film) $v_{\max } 2952,2863,1655,1447,660 \mathrm{~cm}^{-1}$; ${ }^{1} \mathrm{H}$ NMR $\left(200 \mathrm{MHz}, \mathrm{CDCl}_{3}\right) \delta$ 0.91-1.81 (m, $\left.6 \mathrm{H}\right), 1.23$ (s, $3 \mathrm{H}), 1.55$ (s, $3 \mathrm{H}), 2.36-2.60$ (m, $3 \mathrm{H}), 3.55$ (t, $J=7.6 \mathrm{~Hz}, 2 \mathrm{H}) \mathrm{ppm} ;{ }^{13} \mathrm{C}$ NMR/DEPT (50 MHz, $\left.\mathrm{CDCl}_{3}\right) \delta 9.01(\mathrm{q}), 17.5(\mathrm{q}), 29.1(\mathrm{t}), 31.2(\mathrm{t}), 32.6(\mathrm{t}), 43.5(\mathrm{t}), 44.6(\mathrm{~d}), 51.8(\mathrm{~s}), 53.3(\mathrm{t}), 136.5(\mathrm{~s})$, 141.0 (s) ppm; LRMS (EI) m/z 184 (M+1, 5\%), 156 (32), 107 (100), 91 (30).

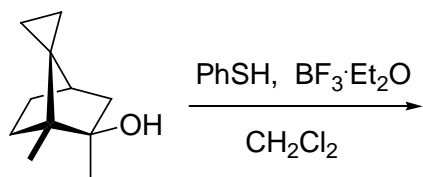

$3 a$

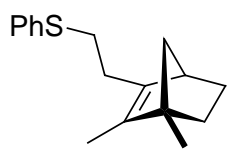

12

Preparation of sulfide 12a" $(X=\mathbf{S P h}, \mathbf{R}=\mathbf{M e})$. - To the mixture of spiral-cyclopropyl camphor derivative $3 \mathbf{a}(20 \mathrm{mg}, 0.12 \mathrm{mmol})$ in $\mathrm{CH}_{2} \mathrm{Cl}_{2}(1 \mathrm{~mL})$ was added thiophenol $(50 \mu \mathrm{L}, 0.48 \mathrm{mmol})$ at -78 ${ }^{\circ} \mathrm{C}$, the mixture was treated with a catalytic amount of $\mathrm{BF}_{3}$ etherate (ca. $10 \mu \mathrm{l}$ ) at the same temperature and was stirred for $30 \mathrm{~min}$ and monitored by TLC. After the starting material was consumed completely, the reaction mixture was treated with an aqueous $\mathrm{CuSO}_{4}$ to remove the excess thiophenol and extracted with $\mathrm{CH}_{2} \mathrm{Cl}_{2}$. The organic extracts were washed with water, brine, and dried $\left(\mathrm{MgSO}_{4}\right)$. The solvent was removed in vacuum and the residue was purified by chromatography on silica gel (petroleum ether) to give $19 \mathrm{mg}(60 \%)$ of the sulfide 12a"' as a colorless oil. $[\alpha]_{\mathrm{D}}{ }^{18}-32\left(c 0.7, \mathrm{CHCl}_{3}\right)$; IR (film) $v_{\max } 2950,1581,1478,1440,738,690 \mathrm{~cm}^{-1} ;{ }^{1} \mathrm{H}$ NMR $\left(200 \mathrm{MHz}, \mathrm{CDCl}_{3}\right) \delta 1.03-1.56(\mathrm{~m}, 4$ H), 1.22 (s, 3 H), 1.49 (s, 3 H), $1.75-2.40$ (m, 4 H), 2.63 (s, 1 H), 2.98 (t, J = 8.0 Hz, 2 H), 7.19-7.52 (m, $5 \mathrm{H}$ ) ppm; HRMS (ESI) $\mathrm{m} / \mathrm{z}[\mathrm{M}+\mathrm{H}]^{+}$found 259.1516, calcd 259.1515 for $\mathrm{C}_{17} \mathrm{H}_{23} \mathrm{~S}$.

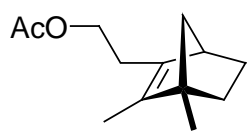

$12 a$

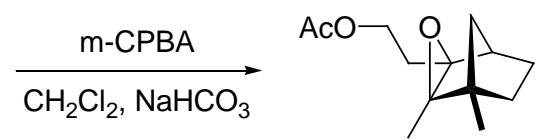

$13 a$

Preparation of norborenyl epoxide 13. - To a solution of the acetate 12a $(86 \mathrm{mg}, 0.41 \mathrm{mmol})$ in $\mathrm{CH}_{2} \mathrm{Cl}_{2}(1.5 \mathrm{~mL})$ was added $\mathrm{m}$-CPBA (153 mg, $\left.0.62 \mathrm{mmol}, 70 \%\right)$ and $\mathrm{NaHCO}_{3}(420 \mathrm{mg}, 5 \mathrm{mmol})$ with stirring at $0{ }^{\circ} \mathrm{C}$. The mixture was stirred for $1 \mathrm{~h}$ and quenched with an aqueous $\mathrm{Na}_{2} \mathrm{SO}_{3}$, extracted with $\mathrm{CH}_{2} \mathrm{Cl}_{2}$ and the organic phases were washed with sat. aqueous $\mathrm{NaHCO}_{3}$, brine, dried over $\mathrm{MgSO}_{4}$, filtered, and concentrated in vacuum. The residue was purified by chromatography to afford $77 \mathrm{mg}$ 
$(85 \%)$ of the exo-epoxide 13a as a colorless oil. 13a $(\mathrm{X}=\mathrm{OAc}, \mathrm{R}=\mathrm{Me}),[\alpha]_{\mathrm{D}}{ }^{21}+33\left(c 1.0, \mathrm{CHCl}_{3}\right)$; IR (film) $v_{\max } 2955,1742,1242,1042 \mathrm{~cm}^{-1} ;{ }^{1} \mathrm{H}$ NMR $\left(200 \mathrm{MHz}, \mathrm{CDCl}_{3}\right) \delta 0.62(\mathrm{~d}, J=9.2 \mathrm{~Hz}, 1 \mathrm{H}), 1.14$ (s, 3 H), 1.19 (s, 3 H), 1.22-1.68 (m, 6 H), 2.07 (s, 3 H), 2.02-2.35 (m, 2 H), 4.28 (t, J = 7.0 Hz, 2 H) ppm; ${ }^{13} \mathrm{C}$ NMR/DEPT (50 MHz, $\left.\mathrm{CDCl}_{3}\right) \delta 8.33$ (q), 15.3 (q), $21.0(\mathrm{q}), 25.3(\mathrm{t}), 26.0(\mathrm{t}), 31.7(\mathrm{t}), 35.6(\mathrm{t})$, 39.7 (d), 46.4 (s), 61.9 (t), 63.7 (s), 64.6 (s), 170.9 (s) ppm; LRMS (EI) m/z 224 (M+1 , 0.04\%), 164 (10), 109 (40), 43 (100); HRMS (ESI) $m / z$ [M+Na] found 247.1309, calcd 247.1305 for $\mathrm{C}_{13} \mathrm{H}_{20} \mathrm{O}_{3} \mathrm{Na}$.

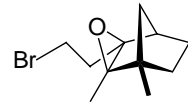

13a' $(\mathrm{X}=\mathrm{Br}, \mathrm{R}=\mathrm{Me})$, colorless oil, $[\alpha]_{\mathrm{D}}{ }^{20}+27\left(\right.$ c $\left.1.0, \mathrm{CHCl}_{3}\right) ;{ }^{1} \mathrm{H}$ NMR $(200 \mathrm{MHz}$, $\left.\mathrm{CDCl}_{3}\right) \delta 0.62(\mathrm{~d}, J=9.4 \mathrm{~Hz}, 1 \mathrm{H}), 1.12(\mathrm{~s}, 3 \mathrm{H}), 1.18(\mathrm{~s}, 3 \mathrm{H}), 1.21-1.34(\mathrm{~m}, 4 \mathrm{H}), 1.58-1.95(\mathrm{~m}, 2 \mathrm{H})$, 2.32-2.48 (m, $2 \mathrm{H}), 3.51$ (t, $J=9.0 \mathrm{~Hz}, 2 \mathrm{H}) \mathrm{ppm} ;{ }^{13} \mathrm{C} \mathrm{NMR} / \mathrm{DEPT}\left(50 \mathrm{MHz}, \mathrm{CDCl}_{3}\right) \delta 8.41$ (q), 15.3 (q), $26.0(\mathrm{t}), 28.8(\mathrm{t}), 30.2(\mathrm{t}), 31.6(\mathrm{t}), 35.5(\mathrm{t}), 39.1(\mathrm{~d}), 46.4(\mathrm{~s}), 64.1$ (s), $65.8(\mathrm{~s})$ ppm; LRMS (EI) m/z $244\left(\mathrm{M}^{+}, 0.4 \%\right), 215$ (13), 137 (27), 109 (100), 91 (14), 79 (19).

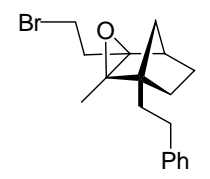

13d $\left(\mathrm{X}=\mathrm{Br}, \mathrm{R}=\right.$ phenethyl), colorless oil, $[\alpha]_{\mathrm{D}}{ }^{21}+36\left(\right.$ c 1.9, $\left.\mathrm{CHCl}_{3}\right) ;{ }^{1} \mathrm{H}$ NMR $(300$ $\left.\mathrm{MHz}, \mathrm{CDCl}_{3}\right) \delta 0.62(\mathrm{~d}, J=9.0 \mathrm{~Hz}, 1 \mathrm{H}), 1.25(\mathrm{~s}, 3 \mathrm{H}), 1.27-1.96(\mathrm{~m}, 8 \mathrm{H}), 2.38-2.48(\mathrm{~m}, 2 \mathrm{H}), 2.67$ (t, $J=10 \mathrm{~Hz}, 2 \mathrm{H}), 3.50-3.57(\mathrm{~m}, 2 \mathrm{H}), 7.15-7.31(\mathrm{~m}, 5 \mathrm{H}) \mathrm{ppm} ;{ }^{13} \mathrm{C}$ NMR/DEPT $\left(75 \mathrm{MHz}, \mathrm{CDCl}_{3}\right) \delta$ $8.79(\mathrm{q}), 25.7(\mathrm{t}), 28.8(\mathrm{t}), 30.1(\mathrm{t}), 30.1(\mathrm{t}), 32.1(\mathrm{t}), 32.1(\mathrm{t}), 32.8(\mathrm{t}), 39.0(\mathrm{~d}), 50.5(\mathrm{~s}), 63.6(\mathrm{~s}), 65.0$ (s), 125.7 (d), 128.1 (d), 128.1 (d), 128.3 (d), 128.3 (d), 142.8 (s) ppm; LRMS (EI) m/z 334 (M+1, 0.3\%), 215 (7), 135 (19), 118 (55), 107 (93), 91 (100), 77 (20), 43 (61); HRMS (ESI) m/z [M+H] found 352.1274, calcd 352.1271 for $\mathrm{C}_{18} \mathrm{H}_{24} \mathrm{OBr}$.

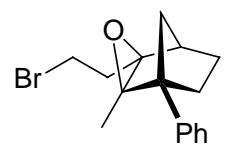

13b $(\mathrm{X}=\mathrm{Br}, \mathrm{R}=\mathrm{Ph})$, crystalline solids, mp. $56-57^{\circ} \mathrm{C}(n-$ Hexane $),[\alpha]_{\mathrm{D}}{ }^{25}+65$ (c 1.1, $\mathrm{CHCl}_{3}$ ); IR (film) $v_{\max } 2958,1601,1445,759,670 \mathrm{~cm}^{-1} ;{ }^{1} \mathrm{H} \mathrm{NMR}\left(300 \mathrm{MHz}, \mathrm{CDCl}_{3}\right) \delta 0.96(\mathrm{~d}, J=10$ Hz, 1 H), 1.02 (s, 3 H), 1.50-1.98 (m, 6 H), 2.44-2.54 (m, 1 H), 2.62 (brs, 1 H), 3.54-3.60 (m, 2 H), 7.24-7.34 (m, $5 \mathrm{H}$ ) ppm; ${ }^{13} \mathrm{C}$ NMR/DEPT (75 MHz, $\mathrm{CDCl}_{3}$ ) $\delta 9.3$ (q), 25.8 (t), $28.0(\mathrm{t}), 28.7(\mathrm{t}), 30.2$ $(\mathrm{t}), 36.1(\mathrm{t}), 39.4(\mathrm{~d}), 54.5(\mathrm{~s}), 65.2(\mathrm{~s}), 65.4(\mathrm{~s}), 126.5(\mathrm{~d}), 127.2(\mathrm{~d}), 127.2(\mathrm{~d}), 128.2(\mathrm{~d}), 128.2(\mathrm{~d})$, 141.0 (s) ppm; HRMS (ESI) $\mathrm{m} / \mathrm{z}[\mathrm{M}+\mathrm{H}]^{+}$found 307.0691, calcd 307.0692 for $\mathrm{C}_{16} \mathrm{H}_{20} \mathrm{OBr}$. 


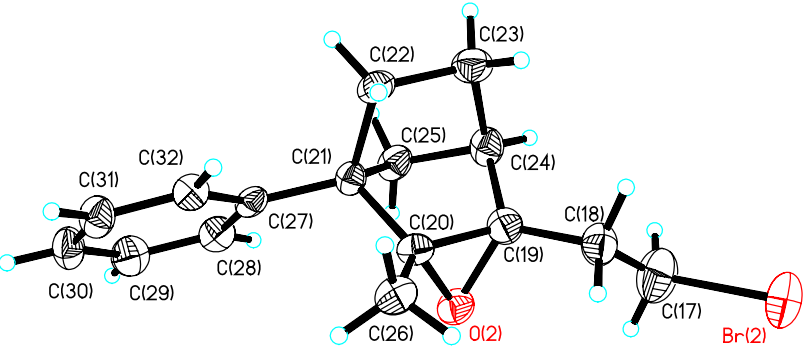

Table 1. Crystal data and structure refinement for epoxide $\mathbf{1 3 b}(X=B r, R=P h)$.

Empirical formula

Formula weight

Temperature

Wavelength

Crystal system

Space group

Unit cell dimensions

Volume

$\mathrm{Z}$

Density (calculated)

Absorption coefficient

$\mathrm{F}(000)$

Crystal size

Theta range for data collection

Index ranges

Reflections collected

Independent reflections

Completeness to theta $=27.88^{\circ}$

Absorption correction

Refinement method

Data / restraints / parameters

Goodness-of-fit on $\mathrm{F}^{2}$

Final $\mathrm{R}$ indices [I $>2 \operatorname{sigma}(\mathrm{I})]$

$\mathrm{R}$ indices (all data)

Absolute structure parameter

Largest diff. peak and hole
$\mathrm{C}_{16} \mathrm{H}_{19} \mathrm{BrO}$

307.22

213(2) K

$0.71073 \AA$

Orthorhombic

P2(1)2(1)2(1)

$\mathrm{a}=8.9507(8) \AA$

$\alpha=90^{\circ}$.

$\mathrm{b}=25.614(2) \AA$

$\beta=90^{\circ}$.

$\mathrm{c}=30.160(3) \AA$

$\gamma=90^{\circ}$.

6914.4(11) $\AA^{3}$

20

$1.476 \mathrm{Mg} / \mathrm{m}^{3}$

$2.959 \mathrm{~mm}^{-1}$

3160

$0.10 \times 0.08 \times 0.08 \mathrm{~mm}^{3}$

1.35 to $27.88^{\circ}$.

$-11<=\mathrm{h}<=11,-33<=\mathrm{k}<=29,-37<=1<=39$

45643

$16373[\mathrm{R}(\mathrm{int})=0.0643]$

$99.7 \%$

None

Full-matrix least-squares on $\mathrm{F}^{2}$

$16373 / 0 / 816$

0.908

$\mathrm{R} 1=0.0427, \mathrm{wR} 2=0.0541$

$\mathrm{R} 1=0.1065, \mathrm{wR} 2=0.0628$

$-0.018(5)$

0.730 and -0.607 e. $\AA^{-3}$ 


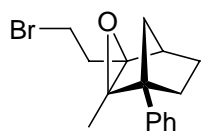

$13 b$

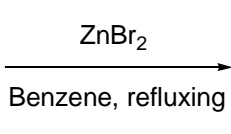

Benzene, refluxing

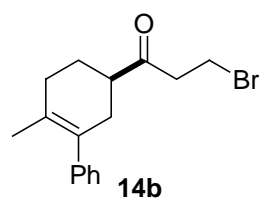

Meinwald rearrangement of 13 to 14 . - To a solution of the epoxide $13(35 \mathrm{mg}, 0.11 \mathrm{mmol})$ in benzene $(3 \mathrm{~mL})$ was added anhydrous zinc bromide $(117 \mathrm{mg}, 0.52 \mathrm{mmol})$, and the mixture was stirred at $70{ }^{\circ} \mathrm{C}$ for about $10 \mathrm{~min}$. After cooling to room temperature, the reaction mixture was diluted with ether, washed with water, brine, and dried over $\mathrm{MgSO}_{4}$. The solvent was removed in vacuum and the residue was purified by chromatography on silica gel to give $27 \mathrm{mg}(80 \%)$ of ketone $14 \mathbf{b}(\mathrm{X}=\mathrm{Br}, \mathrm{R}=$ $\mathrm{Ph})$ as a colorless oil, $[\alpha]_{\mathrm{D}}{ }^{25}+48\left(c\right.$ 1.6, $\left.\mathrm{CHCl}_{3}\right)$; IR (film) $v_{\max } 2914,1711$ (vs), 1440, 1073, 762, 702 $\mathrm{cm}^{-1} ;{ }^{1} \mathrm{H}$ NMR (300 MHz, $\left.\mathrm{CDCl}_{3}\right) \delta 1.56(\mathrm{~s}, 3 \mathrm{H}), 1.65-2.50$ (m, $\left.6 \mathrm{H}\right), 2.69-2.79$ (m, $\left.1 \mathrm{H}\right), 3.04-3.14$ (m, $2 \mathrm{H}), 3.58(\mathrm{t}, J=6.9 \mathrm{~Hz}, 2 \mathrm{H}), 7.13-7.35(\mathrm{~m}, 5 \mathrm{H}) \mathrm{ppm} ;{ }^{13} \mathrm{C}$ NMR/DEPT $\left(75 \mathrm{MHz}, \mathrm{CDCl}_{3}\right) \delta 20.3$ (q), $24.6(t), 25.5(t), 30.9(t), 33.0(t), 43.3(t), 47.6(d), 126.3(d), 128.1(d), 128.1(d), 128.4(d), 128.4$ (d), 128.9 (s), 130.4 (s), 143.2 (s), 209.9 (s) ppm; HRMS (ESI) m/z [M+Na $]^{+}$found 329.0514, calcd 329.0511 for $\mathrm{C}_{16} \mathrm{H}_{19} \mathrm{OBrNa}$.<smiles>COCCC(=O)C1CCC(C)=C(C)C1</smiles>

14a $(X=$ OAc, $R=M e)$, colorless oil, $[\alpha]_{D}^{18}+95\left(\right.$ ( $\left.1.0, \mathrm{CHCl}_{3}\right)$; IR (film) $v_{\max }$ 2915, 1741, 1711, 1368, $1239 \mathrm{~cm}^{-1} ;{ }^{1} \mathrm{H}$ NMR $\left(200 \mathrm{MHz}, \mathrm{CDCl}_{3}\right) \delta$ 0.92-2.14 (m, $\left.6 \mathrm{H}\right), 1.62$ (s, $6 \mathrm{H}, 2$ Me), 2.03 (s, $3 \mathrm{H}), 2.52-2.83(\mathrm{~m}, 3 \mathrm{H}), 4.33$ (t, $J=7.0 \mathrm{~Hz}, 2 \mathrm{H}) \mathrm{ppm} ;{ }^{13} \mathrm{C}$ NMR/DEPT $(50 \mathrm{MHz}$, $\left.\mathrm{CDCl}_{3}\right) \delta 18.8(\mathrm{q}), 19.0(\mathrm{q}), 20.9(\mathrm{q}), 25.0(\mathrm{t}), 31.1(\mathrm{t}), 32.8(\mathrm{t}), 39.2(\mathrm{t}), 47.8(\mathrm{~d}), 59.5(\mathrm{t}), 123.8(\mathrm{~s})$, 125.4 (s), 171.0 (s), 210.5 (s) ppm; HRMS (ESI) $\mathrm{m} / \mathrm{z}[\mathrm{M}+\mathrm{Na}]^{+}$found 247.1308, calcd 247.1305 for $\mathrm{C}_{13} \mathrm{H}_{20} \mathrm{O}_{3} \mathrm{Na}$.<smiles>CC1=C(C)CC(CCCBr)CC1</smiles>

14a' $(\mathrm{X}=\mathrm{Br}, \mathrm{R}=\mathrm{Me})$, colorless oil, $[\alpha]_{\mathrm{D}}{ }^{18}+73\left(\right.$ ( 1.2, $\left.\mathrm{CHCl}_{3}\right)$; IR (film) $v_{\max } 2917$, 1711, 1444, 1381, $911 \mathrm{~cm}^{-1}$; ${ }^{1} \mathrm{H}$ NMR (200 MHz, $\left.\mathrm{CDCl}_{3}\right) \delta 1.62$ (s, $\left.6 \mathrm{H}, 2 \mathrm{Me}\right), 1.89-2.23(\mathrm{~m}, 5 \mathrm{H})$, 2.51-2.56 (m, $1 \mathrm{H}), 3.06$ (t, $J=6.8 \mathrm{~Hz}, 2 \mathrm{H}$ ), 3.57 (t, $J=6.9 \mathrm{~Hz}, 2 \mathrm{H}$ ) ppm; HRMS (ESI) $\mathrm{m} / z[\mathrm{M}+\mathrm{H}]^{+}$ found 245.0537, calcd 245.0536 for $\mathrm{C}_{11} \mathrm{H}_{18} \mathrm{OBr}$.<smiles>CC1=C(CCc2ccccc2)CC(C(=O)CCBr)CC1</smiles>

14d $\left(X=B r, R=\right.$ phenethyl), colorless oil, $[\alpha]_{D}^{21}+55\left(\right.$ c 1.8, $\left.\mathrm{CHCl}_{3}\right)$; IR (film) $v_{\max }$ 
2921, 2856, 1710, 1452, 1075, 909, 733, $700 \mathrm{~cm}^{-1} ;{ }^{1} \mathrm{H}$ NMR (300 MHz, $\left.\mathrm{CDCl}_{3}\right) \delta 1.54(\mathrm{~s}, 3 \mathrm{H})$, 1.49-2.32 (m, 8 H), 2.50-2.71 (m, 3 H), 3.00-3.14 (m, 2 H), 3.57 (t, J = 6.7 Hz, 2 H), 7.16-7.30 (m, 5 H) ppm; ${ }^{13} \mathrm{C} \mathrm{NMR/DEPT}\left(75 \mathrm{MHz}, \mathrm{CDCl}_{3}\right) \delta 18.5(\mathrm{q}), 24.9(\mathrm{t}), 25.6(\mathrm{t}), 31.0(\mathrm{t}), 31.2(\mathrm{t}), 34.5(\mathrm{t}), 35.5$ (t), $43.3(\mathrm{t}), 47.7$ (d), 125.8 (d), 126.7 (s), 127.4 (s), 128.2 (d), 128.2 (d), 128.4 (d), 128.4 (d), 142.2 (s), 210.4 (s) ppm; LRMS (EI) m/z 334 (M+, 3\%), 199 (12), 165 (9), 137 (31), 107 (100), 91 (96), 77 (21), 65 (26); HRMS (ESI) $m / z[\mathrm{M}+\mathrm{H}]^{+}$found 352.1273, calcd 352.1271 for $\mathrm{C}_{18} \mathrm{H}_{24} \mathrm{OBr}$.

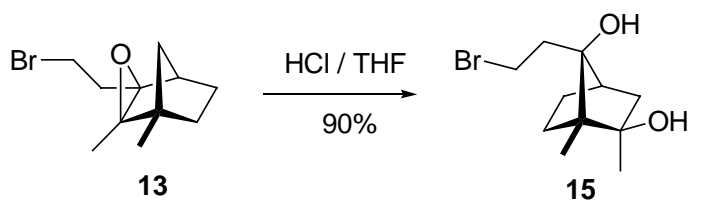

Preparation of bicyclic diol 15 from epoxide 13. - A solution of the epoxide 13 ( $24 \mathrm{mg}, 0.1 \mathrm{mmol})$ in $0.5 \mathrm{~mL}$ of THF was treated dropwise with $0.5 \mathrm{~mL}$ of $20 \% \mathrm{HCl}$ at room temperature. The reaction mixture was stirred for 25 minutes and quenched with powdered $\mathrm{NaHCO}_{3}$, and extracted with EtOAc $(15 \mathrm{~mL})$. Combined extracts were washed with water, dried over $\mathrm{MgSO}_{4}$, filtered, and concentrated. The residue was purified by chromatography on silica gel to afford $23 \mathrm{mg}(90 \%)$ of diol 15 as crystalline solids, mp. $135-136^{\circ} \mathrm{C}$ (ethyl acetate); $[\alpha]_{\mathrm{D}}{ }^{20}+33$ (c 1.0, $\mathrm{CHCl}_{3}$ ); IR (film) $v_{\max } 3356,3194$, 2962, $1448 \mathrm{~cm}^{-1}$; ${ }^{1} \mathrm{H}$ NMR $\left(200 \mathrm{MHz}, \mathrm{CDCl}_{3}\right) \delta 0.94(\mathrm{~s}, 3 \mathrm{H}), 1.28(\mathrm{~s}, 3 \mathrm{H}), 0.85-1.60(\mathrm{~m}, 6 \mathrm{H})$, 2.07-2.16 (m, $3 \mathrm{H}), 2.61$ (s, $1 \mathrm{H}), 3.46-3.71$ (m, $2 \mathrm{H}), 3.97$ (s, $1 \mathrm{H}) \mathrm{ppm} ;{ }^{13} \mathrm{C}$ NMR/DEPT (50 MHz, $\left.\mathrm{CDCl}_{3}\right) \delta 9.53(\mathrm{q}), 24.1(\mathrm{q}), 25.2(\mathrm{t}), 29.2(\mathrm{t}), 29.3(\mathrm{t}), 35.8(\mathrm{t}), 42.3(\mathrm{~d}), 46.7(\mathrm{t}), 51.3(\mathrm{~s}), 80.5(\mathrm{~s}), 88.5$ (s) ppm; LRMS (EI) m/z 244 ([M- $\left.\mathrm{H}_{2} \mathrm{O}\right]^{+}, 4 \%$ ), 186 (15), 137 (24), 109 (100), 93 (21), 43 (36).

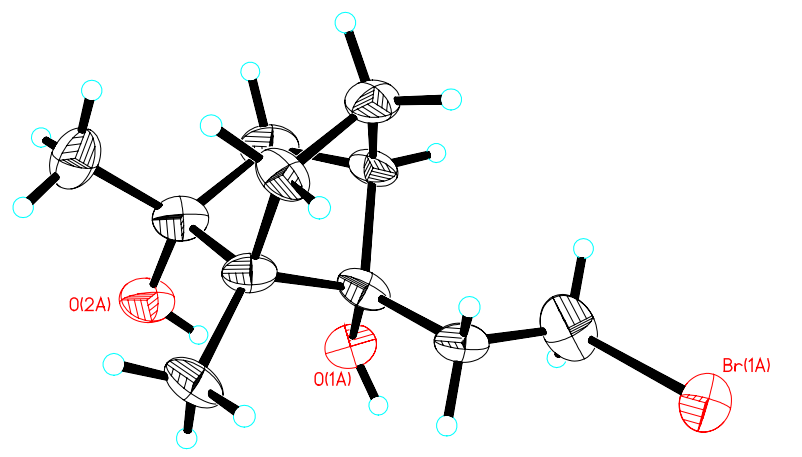


Table 2. Crystal data and structure refinement for diol $\mathbf{1 5 .}$

Empirical formula

Formula weight

Temperature

Wavelength

Crystal system

Space group

Unit cell dimensions

Volume

Z

Density (calculated)

Absorption coefficient

$\mathrm{F}(000)$

Crystal size

Theta range for data collection

Index ranges

Reflections collected

Independent reflections

Completeness to theta $=27.89^{\circ}$

Absorption correction

Max. and min. transmission

Refinement method

Data / restraints / parameters

Goodness-of-fit on $\mathrm{F}^{2}$

Final $\mathrm{R}$ indices [I $>2 \operatorname{sigma}(\mathrm{I})]$

$\mathrm{R}$ indices (all data)

Absolute structure parameter

Largest diff. peak and hole
$\mathrm{C}_{11} \mathrm{H}_{19} \mathrm{BrO}_{2}$

263.17

213(2) K

$0.71073 \AA$

Orthorhombic

P2(1)2(1)2(1)

$\mathrm{a}=7.2349(15) \AA \quad \alpha=90^{\circ}$.

$\mathrm{b}=7.3055(15) \AA \quad \beta=90^{\circ}$.

$\mathrm{c}=44.210(9) \AA \quad \gamma=90^{\circ}$.

2336.7(8) $\AA^{3}$

8

$1.496 \mathrm{Mg} / \mathrm{m}^{3}$

$3.493 \mathrm{~mm}^{-1}$

1088

$0.20 \times 0.18 \times 0.10 \mathrm{~mm}^{3}$

0.92 to $27.89^{\circ}$.

$-9<=\mathrm{h}<=9,-9<=\mathrm{k}<=6,-58<=1<=58$

15105

$5525[\mathrm{R}($ int $)=0.0799]$

$99.2 \%$

Empirical

0.7214 and 0.5417

Full-matrix least-squares on $\mathrm{F}^{2}$

$5525 / 0 / 261$

1.084

$\mathrm{R} 1=0.0647, \mathrm{wR} 2=0.1417$

$\mathrm{R} 1=0.0921, \mathrm{wR} 2=0.1490$

0.061(17)

0.551 and -0.684 e. $\AA^{-3}$

$\gamma_{\mathrm{H}}$

16, colorless oil. ${ }^{6}$ IR (film) $v_{\max } 3449,2950,2866,1451,1364,1074,1006,738 \mathrm{~cm}^{-1}$;

${ }^{1} \mathrm{H}$ NMR (300 MHz, $\left.\mathrm{CDCl}_{3}\right) \delta 0.22-0.83(\mathrm{~m}, 4 \mathrm{H}), 1.14-1.89(\mathrm{~m}, 7 \mathrm{H}), 2.91(\mathrm{~d}, J=3.6 \mathrm{~Hz}, 1 \mathrm{H}, \mathrm{OH})$,

\footnotetext{
${ }^{6}$ Prepared from 10-hydroxy-7-spiral-cyclopropylcamphor by benzylation and carbonyl reduction, cf.: Föhlisch, B.; Bakr, D. A.; Fischer, P. J. Org. Chem. 2002, 67, 3682.
} 
$3.43(\mathrm{q}, J=9.6 \mathrm{~Hz}, 2 \mathrm{H}), 3.96-3.99(\mathrm{~m}, 1 \mathrm{H}), 4.46(\mathrm{q}, J=12 \mathrm{~Hz}, 2 \mathrm{H}), 7.26-7.37(\mathrm{~m}, 5 \mathrm{H}) \mathrm{ppm} ;{ }^{13} \mathrm{C}$ NMR (75 MHz, $\left.\mathrm{CDCl}_{3}\right) \delta$ 0.90, 5.16, 27.9, 30.0, 32.9, 41.4, 43.1, 48.9, 70.2, 73.5, 77.4, 127.4, 127.4, 127.6, 128.4, 128.4, 138.0 ppm; LRMS (EI) m/z 258 (M+1 0.1\%), 167 (2), 150 (26), 106 (32), 91 (100), 79 (23); HRMS (ESI) $m / z$ [2M+H] $]^{+}$found 517.3316; calcd 517.3312 for $\mathrm{C}_{34} \mathrm{H}_{45} \mathrm{O}_{4}$.<smiles>OC1C(O)C2CCC1C2O</smiles>

17, colorless oil. IR (film) $v_{\max }$ 2952, 2867, 1734, 1367, 1246, 1099, 1053, 1018, 740 $\mathrm{cm}^{-1} ;{ }^{1} \mathrm{H}$ NMR $\left(300 \mathrm{MHz}, \mathrm{CDCl}_{3}\right) \delta$ 0.27-0.61 (m, 4H), 1.25-2.02 (m, 7H), 1.91 (s, 3H), 3.12 (d, $J=$ $9.0 \mathrm{~Hz}, 1 \mathrm{H}), 3.38(\mathrm{~d}, J=9.0 \mathrm{~Hz}, 1 \mathrm{H}), 4.41(\mathrm{q}, J=12 \mathrm{~Hz}, 2 \mathrm{H}), 4.86-4.90(\mathrm{~m}, 1 \mathrm{H}), 7.26-7.33(\mathrm{~m}, 5 \mathrm{H})$ ppm; ${ }^{13} \mathrm{C}$ NMR (75 MHz, $\left.\mathrm{CDCl}_{3}\right) \delta$ 2.29, 4.58, 21.2, 27.7, 29.5, 33.5, 39.7, 43.1, 48.5, 68.2, 73.3, 78.3, 127.3, 127.3, 127.3, 128.2, 128.2, 138.7, 170.7 ppm; LRMS (EI) m/z $300\left(\mathrm{M}^{+}, 0.1 \%\right), 209$ (2), 134 (30), 106 (19), 91(100), 79 (12); HRMS (SMS) m/z [M+H] found 301.1792; calcd 301.1798 for $\mathrm{C}_{19} \mathrm{H}_{25} \mathrm{O}_{3}$.

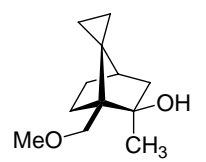

18, colorless oil. ${ }^{7}$ IR (film) $v_{\max }$ 3496, 2934, 2870, 1187, $1103 \mathrm{~cm}^{-1} ;{ }^{1} \mathrm{H}$ NMR (300 $\left.\mathrm{MHz}, \mathrm{CDCl}_{3}\right) \delta$ 0.24-0.92 (m, 4H), 1.14-1.96 (m, 8H), $1.34(\mathrm{~s}, 3 \mathrm{H}), 3.26(\mathrm{~s}, 3 \mathrm{H}), 3.32-3.41(\mathrm{~m}, 2 \mathrm{H})$ ppm; ${ }^{13} \mathrm{C}$ NMR $\left(75 \mathrm{MHz}, \mathrm{CDCl}_{3}\right) \delta 1.7,6.4,24.3,27.9,28.3,35.5,43.3,49.2,51.4,59.4,72.3,80.3$ ppm; LRMS (EI) m/z 164 ([M-32] , 4\%), 149 (14), 121 (26), 106 (100), 91 (62).

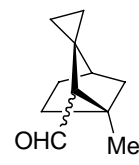

19, colorless oil (mixture of inseparable isomers, ca 1:1), prepared from methyl ether $\mathbf{1 8}$ by acidolysis with catalytic amount of $p$ - $\mathrm{TsOH}$ in refluxing benzene in $80 \%$ yield. IR (film) $v_{\max } 2953$, 2869, 1716, $1669 \mathrm{~cm}^{-1} ;{ }^{1} \mathrm{H}$ NMR $\left(300 \mathrm{MHz}, \mathrm{CDCl}_{3}\right) \delta$ 0.20-0.69 (m, 8H), 1.17 (s, 3H), $1.23(\mathrm{~s}, 3 \mathrm{H})$, $1.25-2.13(\mathrm{~m}, 16 \mathrm{H}), 9.47(\mathrm{~d}, J=6.6 \mathrm{~Hz}, 1 \mathrm{H}), 9.62(\mathrm{~d}, J=5.4 \mathrm{~Hz}, 1 \mathrm{H}) \mathrm{ppm} ;{ }^{13} \mathrm{C} \mathrm{NMR}\left(75 \mathrm{MHz}, \mathrm{CDCl}_{3}\right)$ $\delta 6.0,6.8,11.5,13.4,18.3,20.4,27.6,28.4,28.6,28.9,30.5,37.7,43.4,45.8,46.8,47.3,49.3,51.3$, 62.3, 63.8, 203.7, 206.9 ppm; LRMS (EI) m/z 164 (M+, 7\%), 135 (31), 107 (43), 91 (100), 79 (91).

\footnotetext{
${ }^{7}$ Prepared from 10-hydroxy-7-spiral-cyclopropylcamphor by O-methylation and C(2) carbonyl methylation, cf.: Föhlisch, B.; Bakr, D. A.; Fischer, P. J. Org. Chem. 2002, 67, 3682.
} 

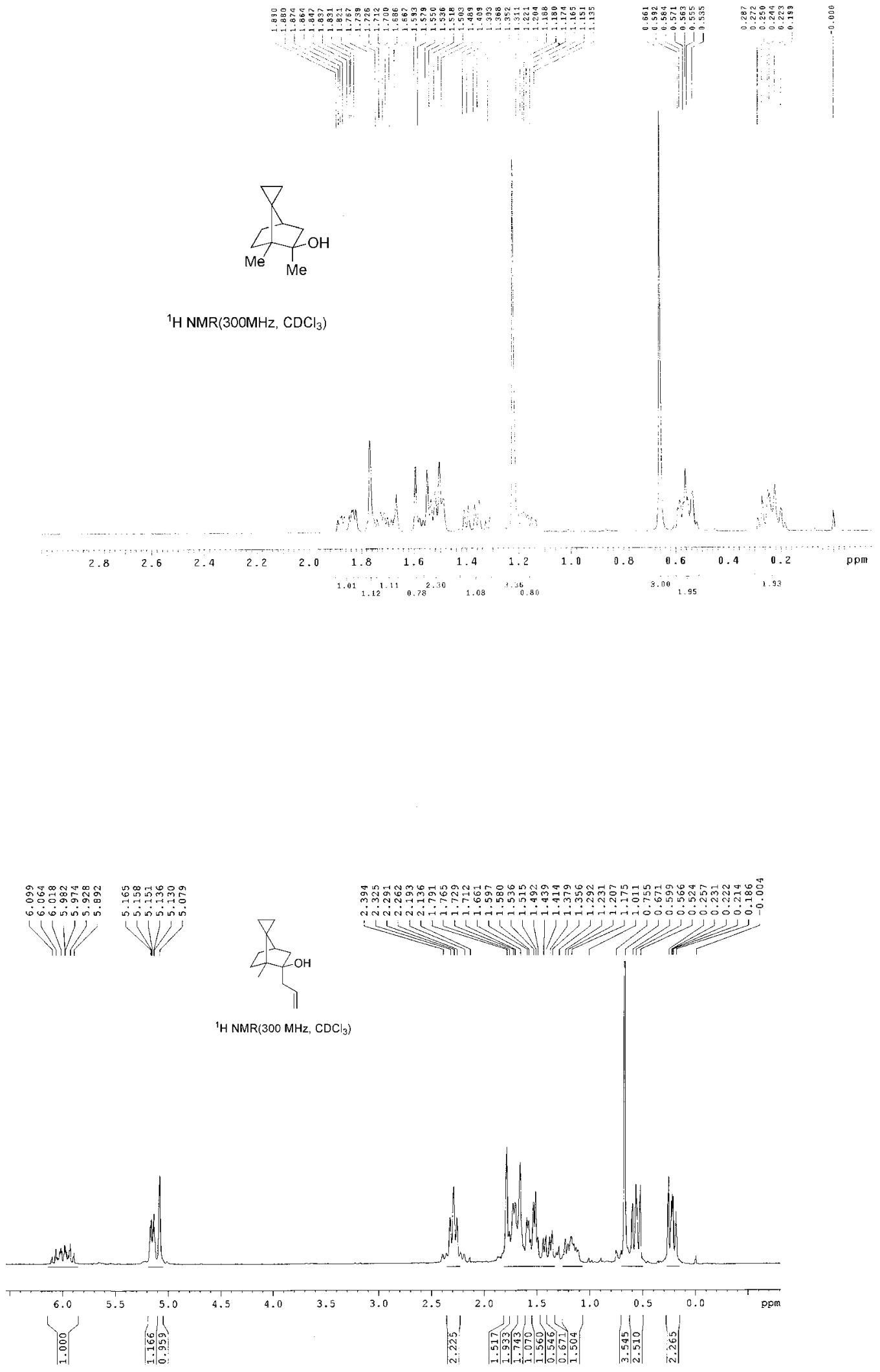


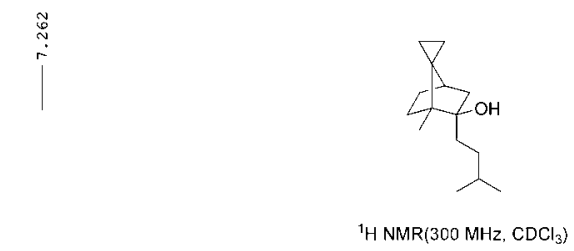

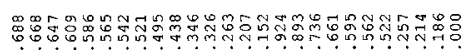

Lic

$\mathrm{NMR}\left(300 \mathrm{MHz}_{2} \mathrm{CDCl}_{3}\right)$
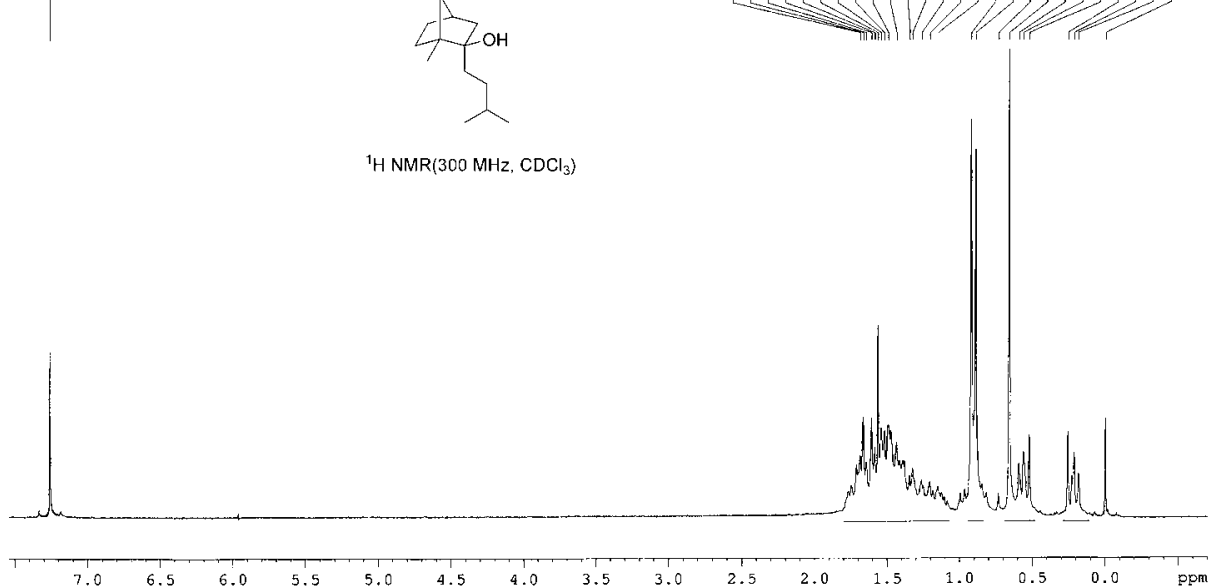

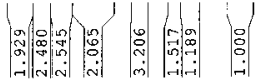
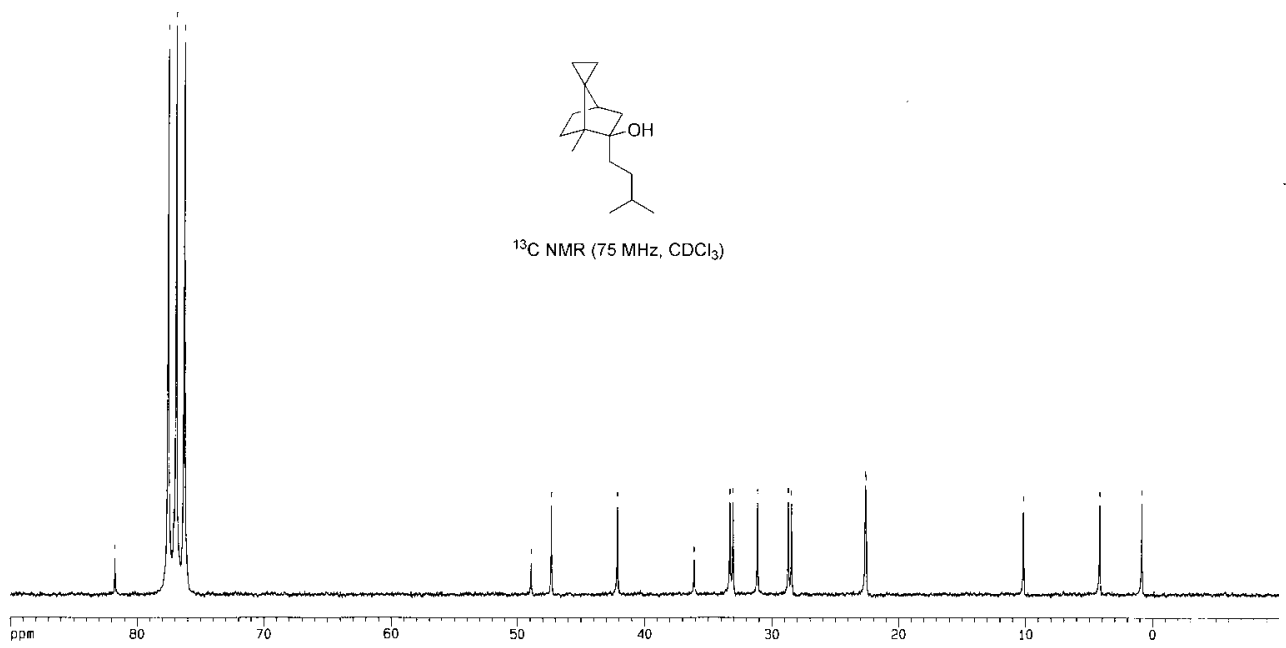


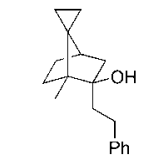

TANG YU RONG

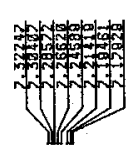

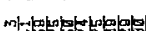

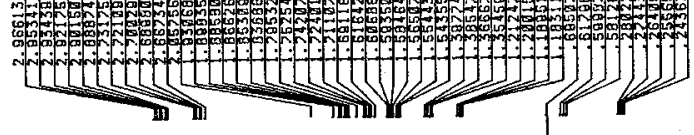

${ }^{1} \mathrm{HNMR}\left(400 \mathrm{MHz}, \mathrm{CDCl}_{3}\right)$
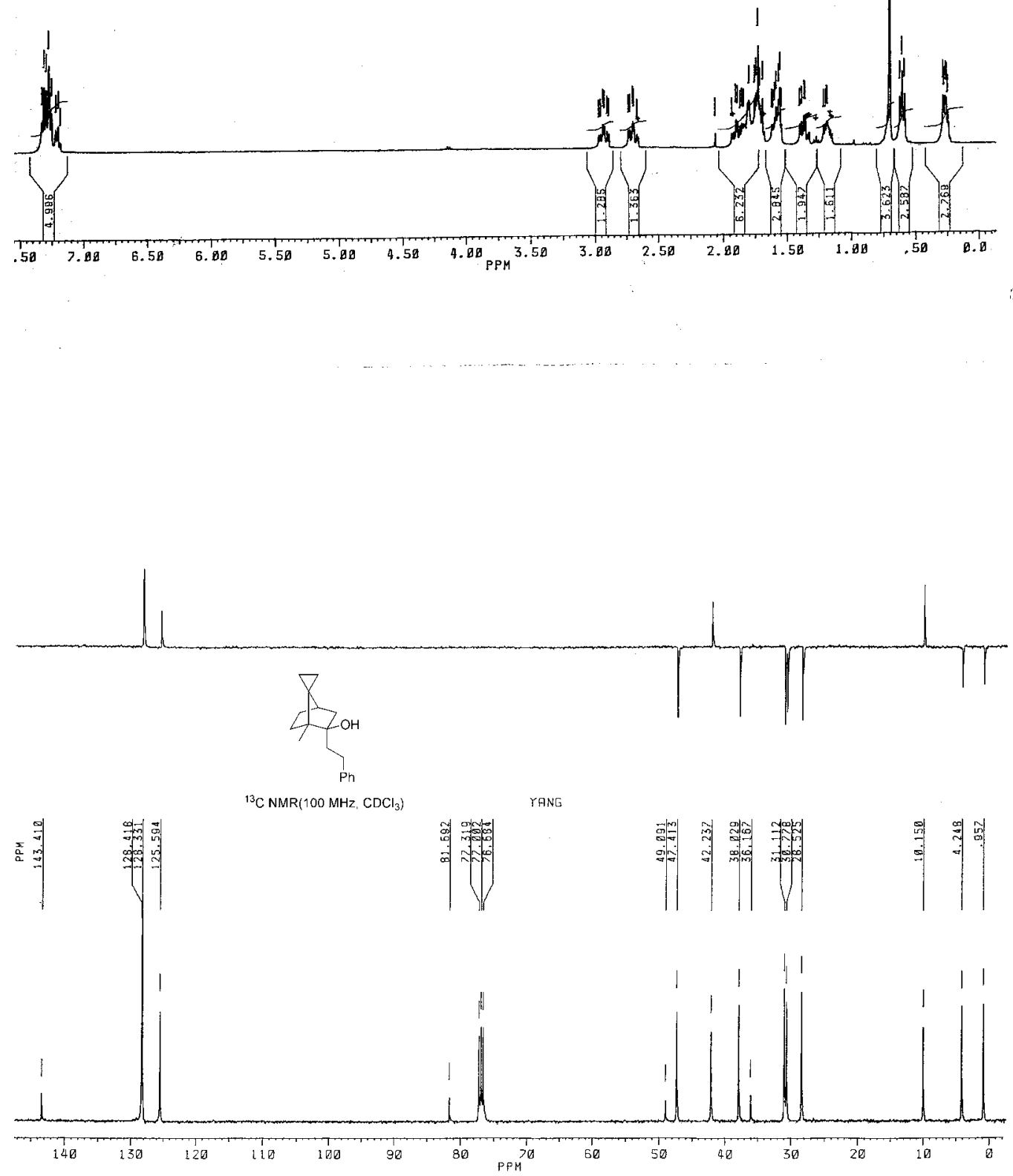

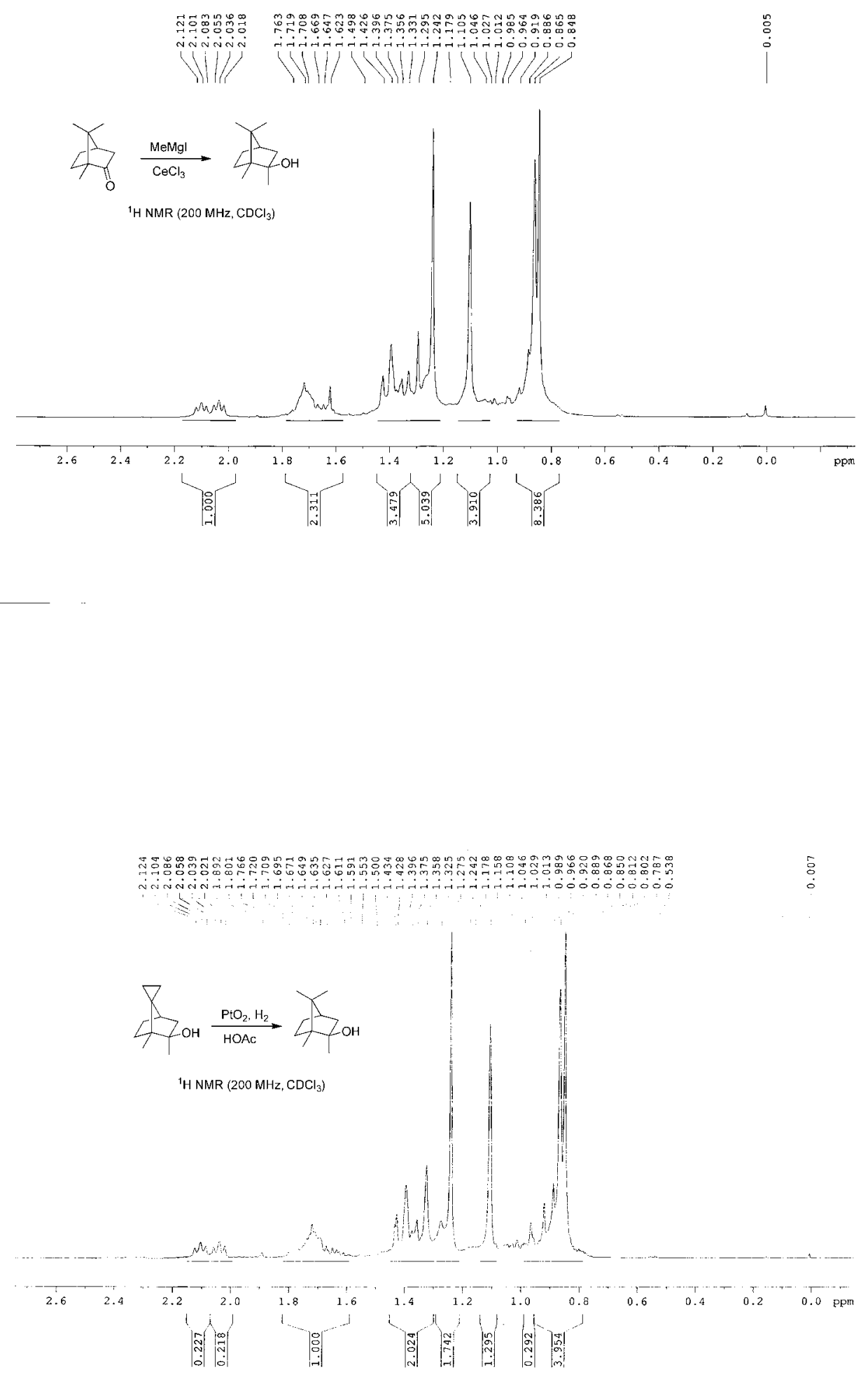
yangyurong

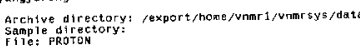

ulse sequence: 52
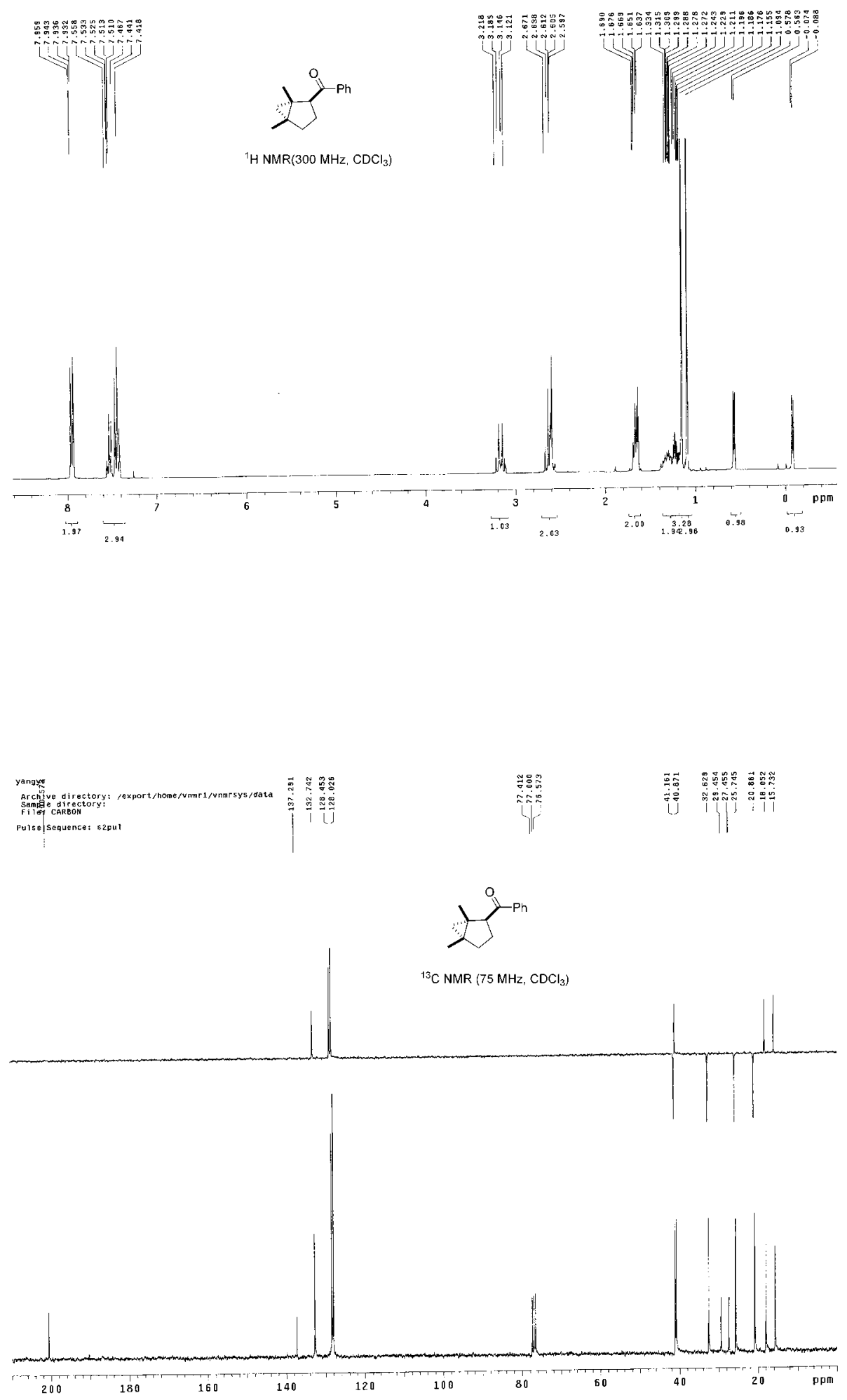


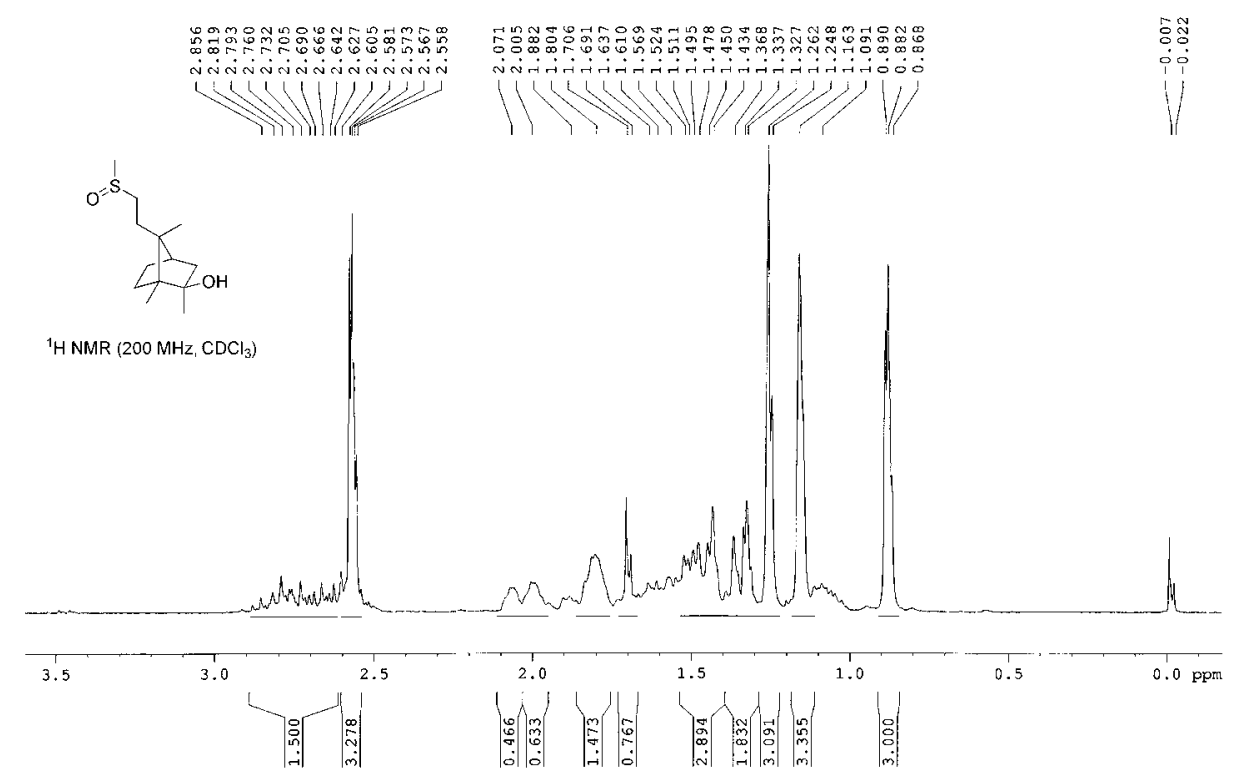

|

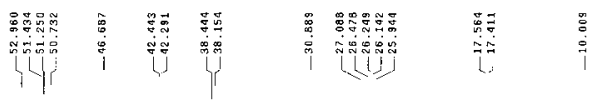

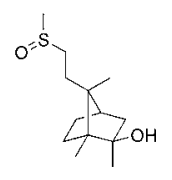

${ }^{13} \mathrm{C}$ NMR (75 $\mathrm{MHz}, \mathrm{CDCl}_{3}$ )

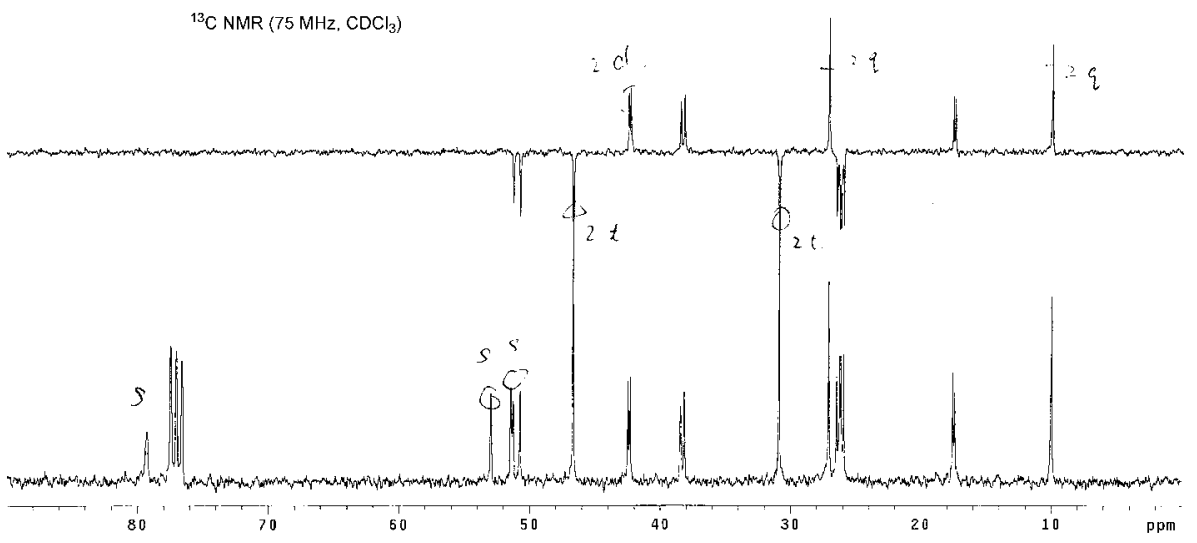



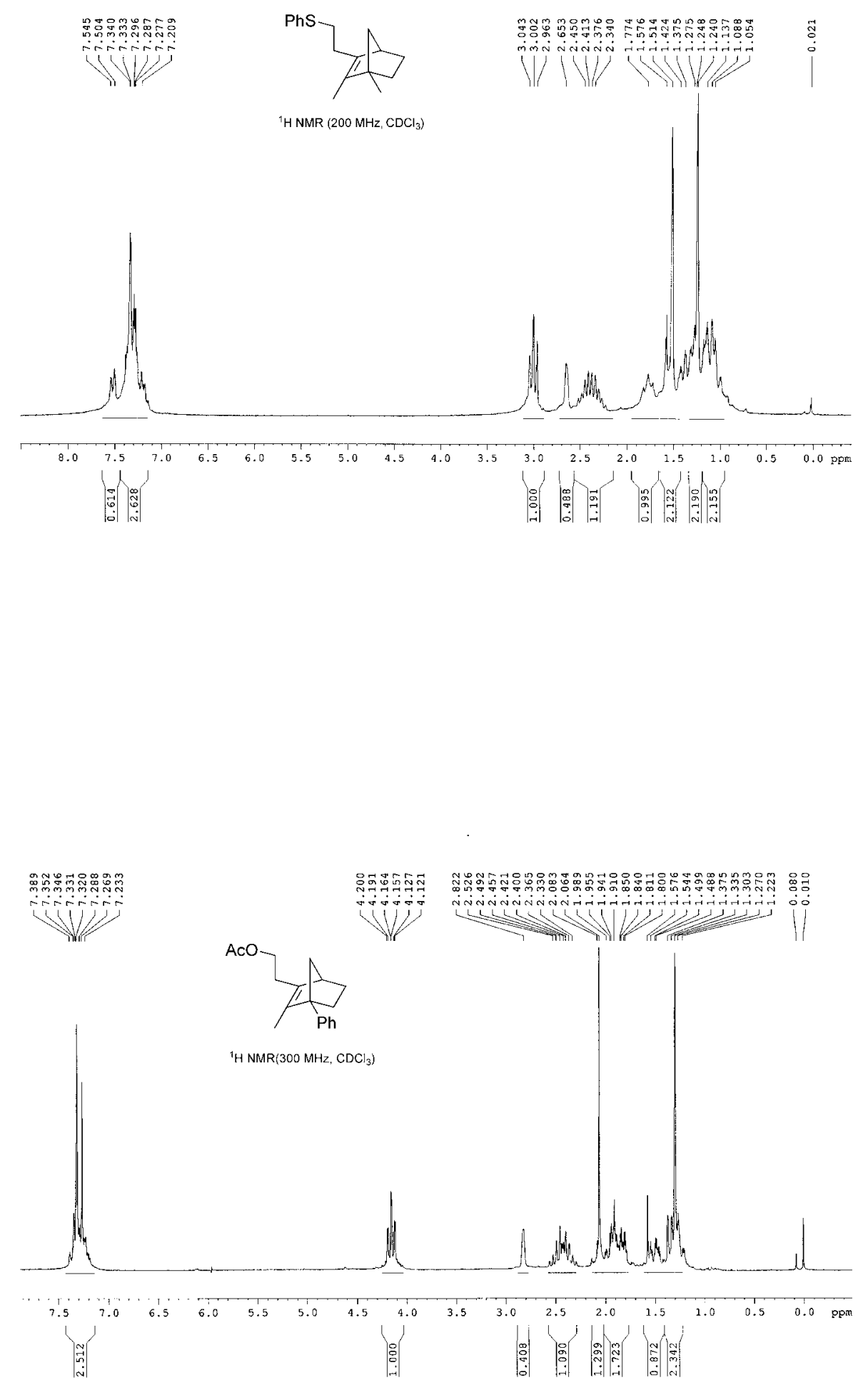

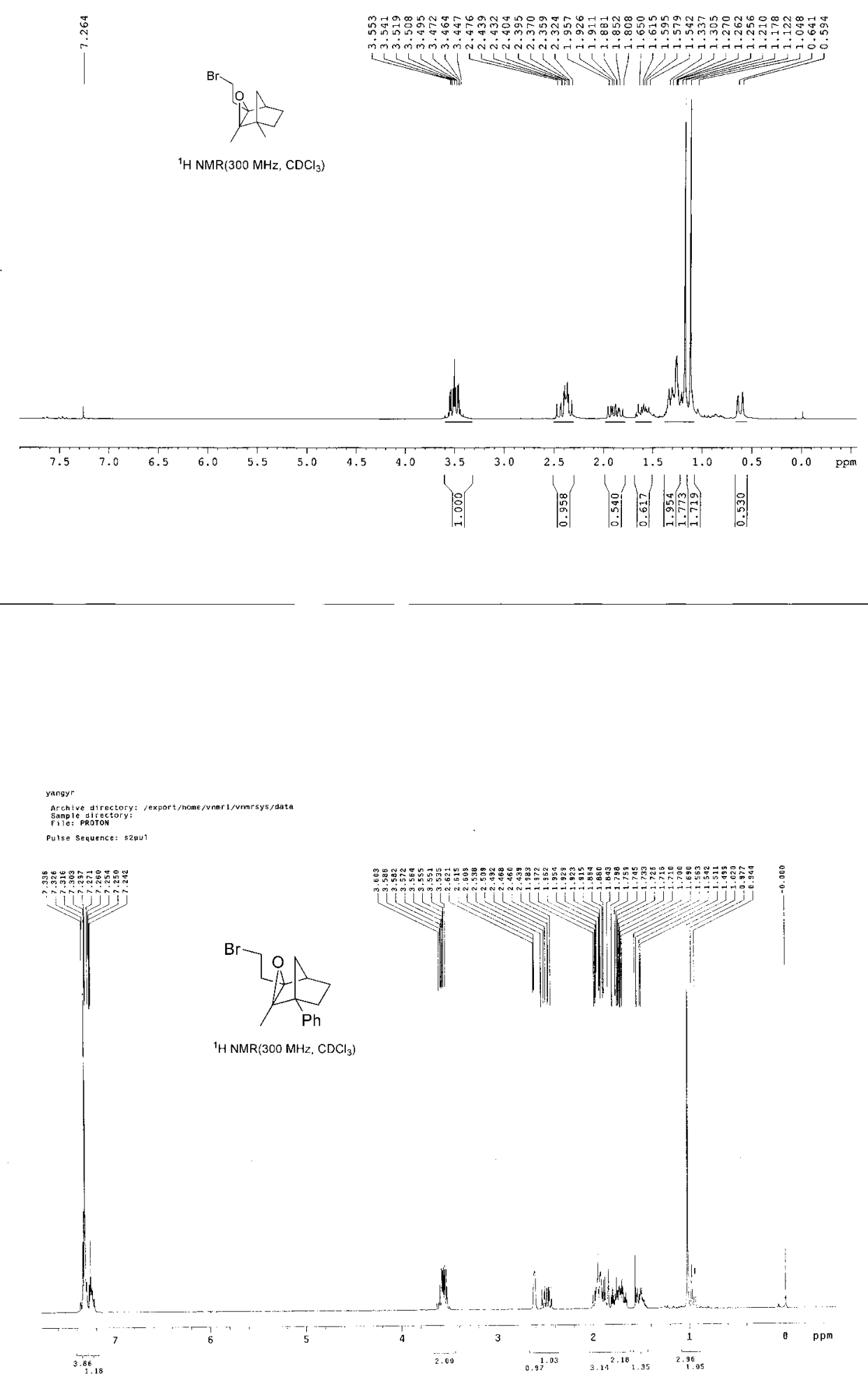


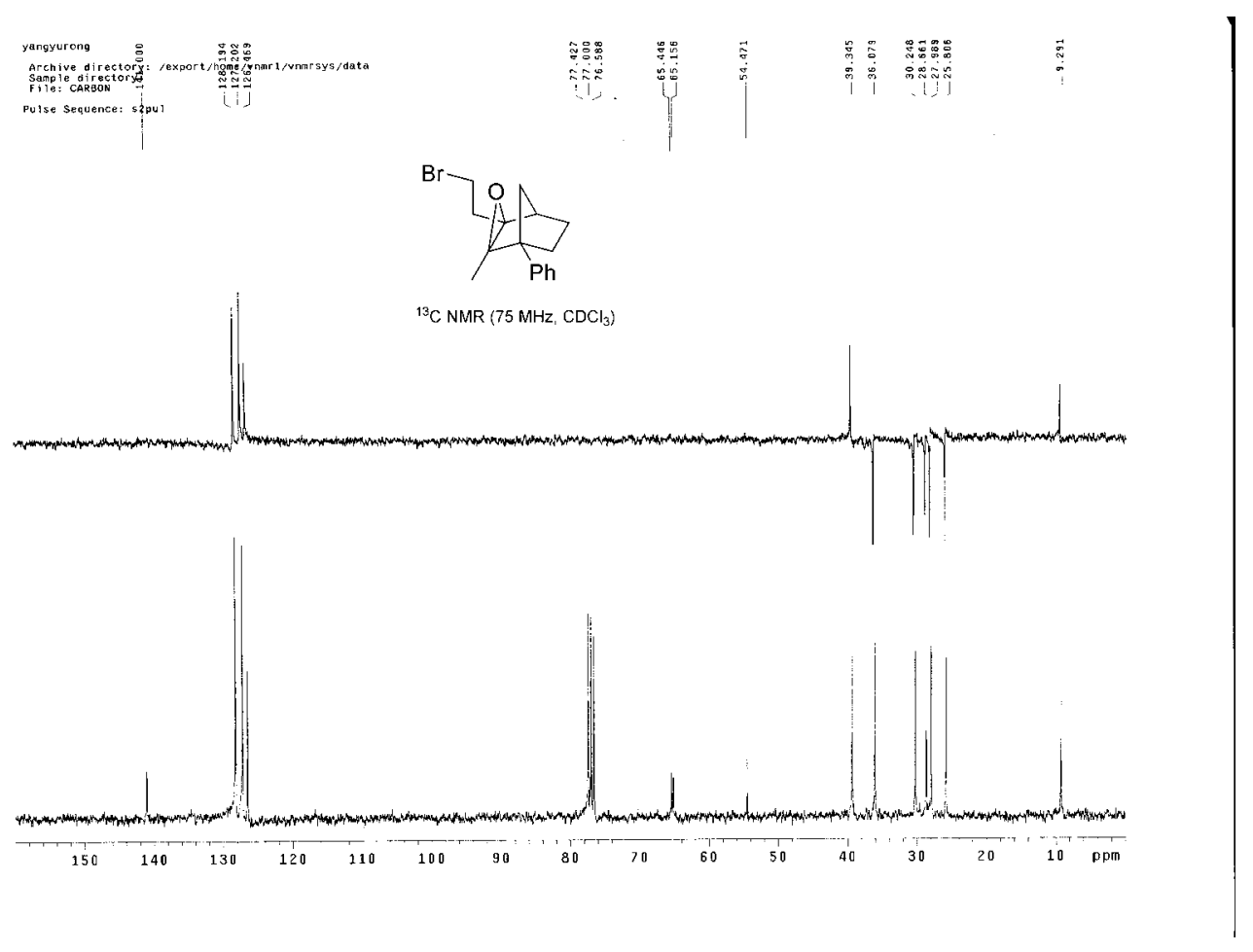

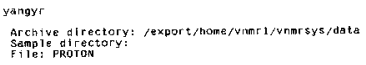

Pules saguance: szzur

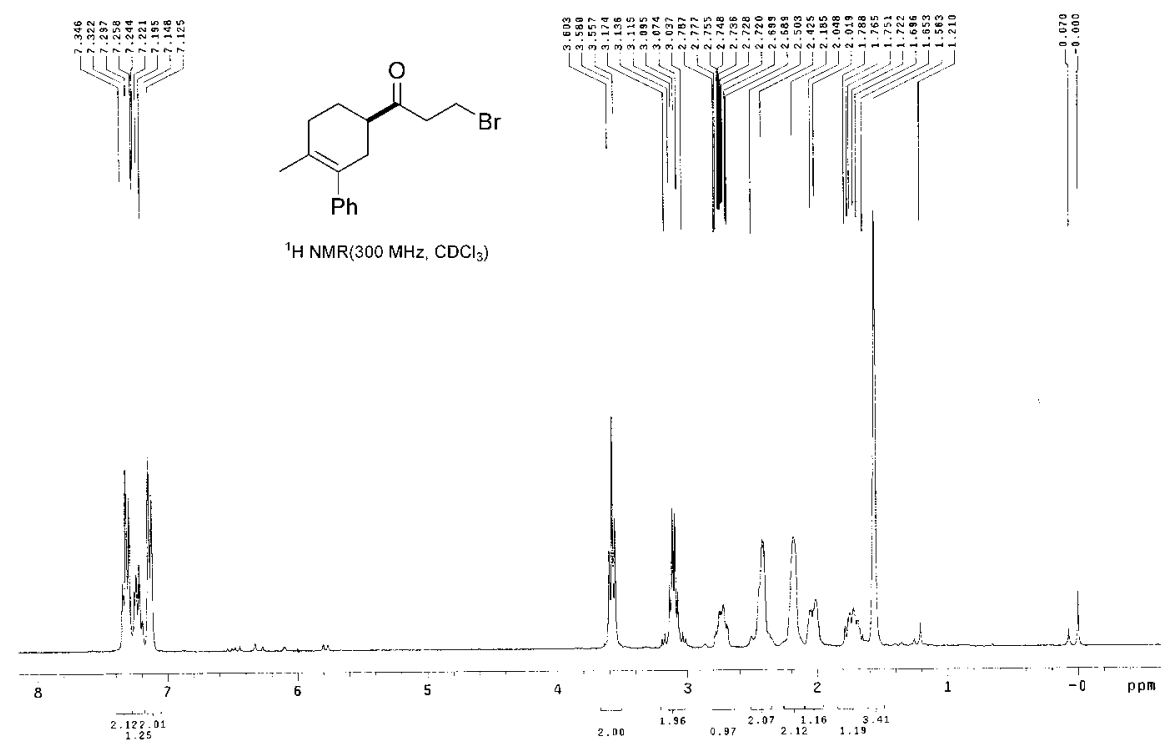




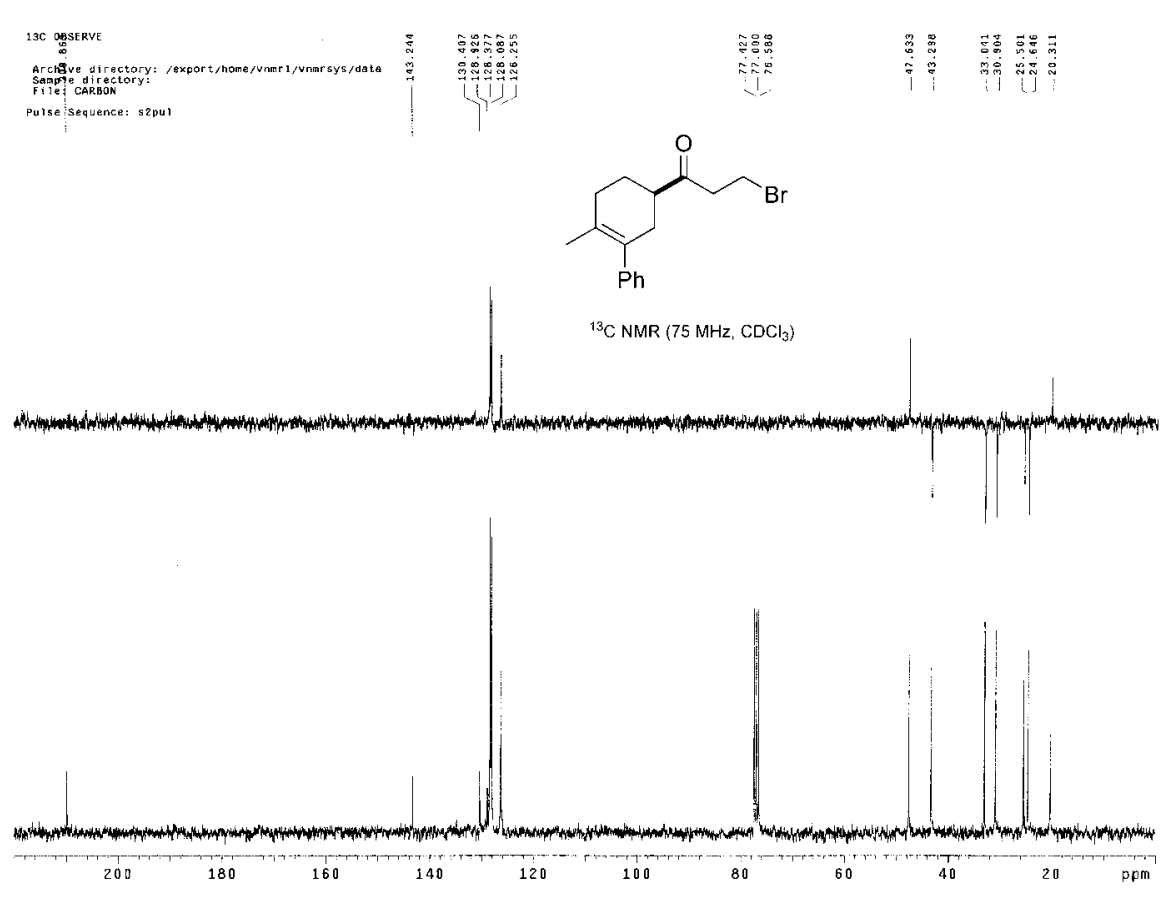

yangyr

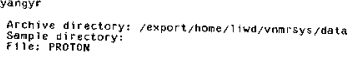

Pulse scauence: s2pul
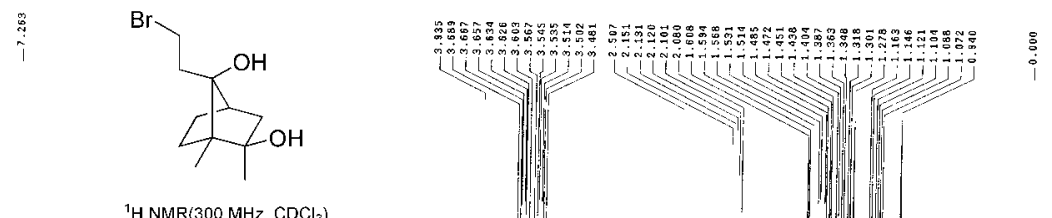

${ }^{1} \mathrm{HNMR}\left(300 \mathrm{MHz}_{1} \mathrm{CDCl}_{3}\right)$

:
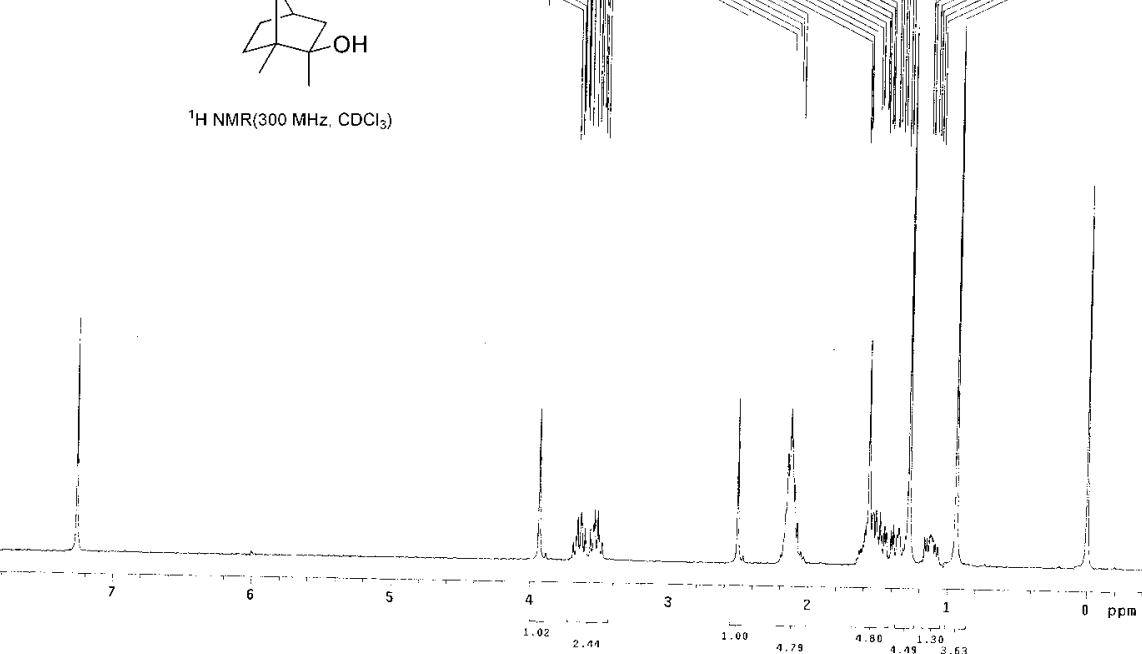

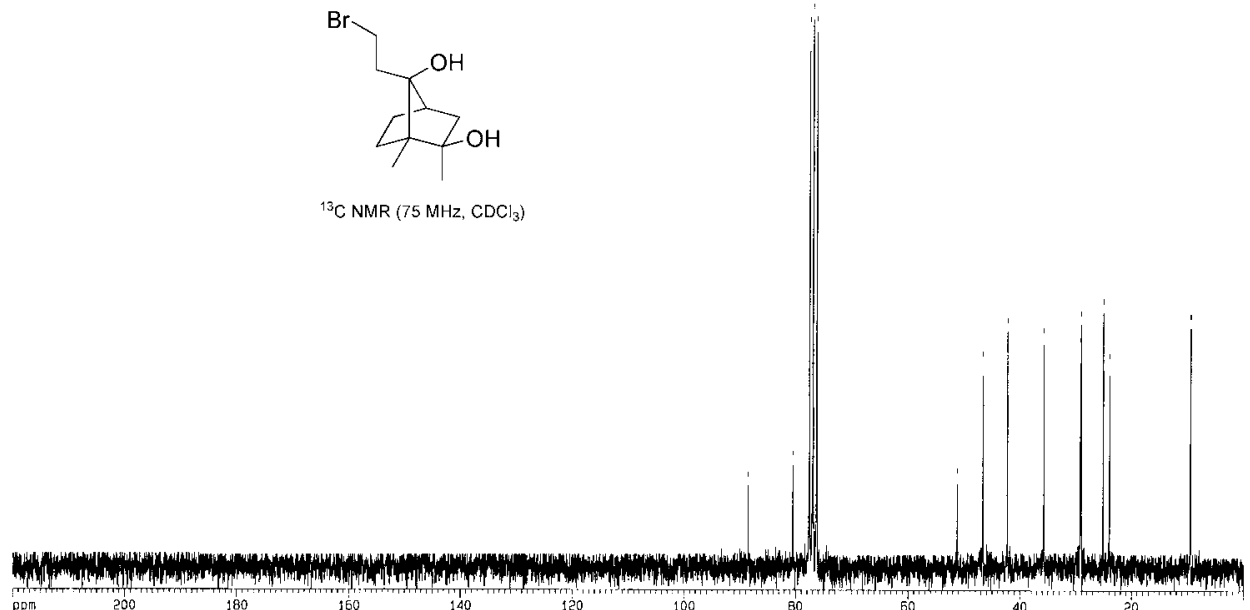

$\mid$

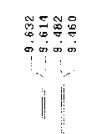
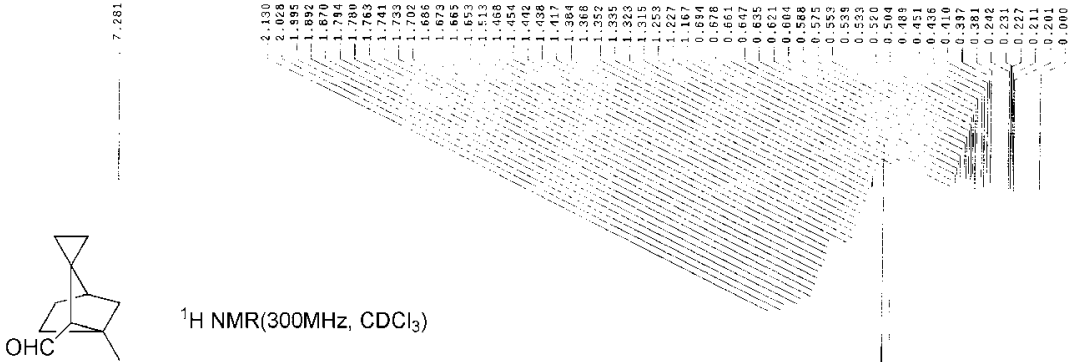

${ }^{1} \mathrm{H} \mathrm{NMR}\left(30 \mathrm{OMHz}, \mathrm{CDCl}_{3}\right)$

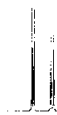

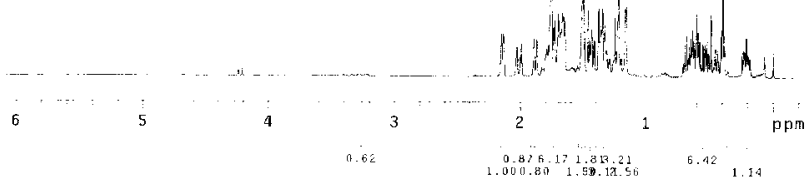

